\title{
Analysis on Riemannian co-compact covers
}

\author{
Laurent Saloff-Coste
}

\begin{abstract}
This is a survey on analysis on non-compact co-compact Riemannian covers and how it relates to random walks on finitely generated groups. The focus is on the long time behavior of the heat kernel and related topics such as Liouville theorems, scale invariant Harnack inequalities and isoperimetric profiles.
\end{abstract}

\section{Contents}

1. Introduction

2. Some quasi-isometric invariants 356

3. Invariance of the heat decay 366

4. The heat decay for random walks 367

5. Harmonic functions 373

$\begin{array}{ll}\text { References } & 380\end{array}$

\section{Introduction}

This article surveys results concerning the large scale potential theory of noncompact co-compact Riemannian regular covers. Here, "large scale potential theory" means the large scale analysis of the Laplace operator and heat equation. This includes heat kernel estimates, Liouville theorems and related topics. The fundamental idea behind most of the results described in this survey is that the large scale potential theory of a co-compact Riemannian cover is determined by the behavior of some basic random walks on its deck transformation group. Thus this article also reviews the relevant results concerning random walks on finitely generated groups. Of course, what is reviewed in this survey reflects in parts the author's personal taste and limitations. For pointers to certain aspects (e.g., Novikov-Shubin invariants) that are not covered here, see $[\mathbf{2 0}, \mathbf{4 1}, \mathbf{8 1}]$.

2000 Mathematics Subject Classification. Primary 20F69,31C12,58J35,60G50

Key words and phrases. co-compact regular covers, heat kernel, random walks, potential theory, isoperimetry.

Research supported in part by NSF Grant \# 0102126. 
1.1. Acknowledgments. This surveys owes a lot to contacts with colleagues and friends over the years. Many thanks to Alano Ancona, Philippe Bougerol, Yves Guivarc'h, V. Kaimanovich, François Ledrappier, Terry Lyons, and Wolfgang Woess. Thanks to T. Coulhon, A. Grigor'yan, W. Hebisch and C. Pittet for sharing their ideas with me. One of the well-known open questions recorded in this survey concerns the stability of the Liouville property, say, on Cayley graphs. I remember very well being introduced to this question and many related ideas present in this survey by N. Varopoulos, just after I finished my Ph.D, twenty years ago.

In August 2003, I sent a first draft of this survey to a few friends, including Martine Babillot, asking for help and comments. I learned that Martine would not respond. She had left us. I miss her.

1.2. Manifolds. Let $(M, g)$ be a non-compact complete Riemannian manifold. Let $\mathcal{C}_{0}^{\infty}(M)$ be the space of smooth compactly supported functions on $M$. Denote by $\Delta$ the Laplace-Beltrami operator and by $\mu$ the Riemannian measure. Thus, in local coordinates,

$$
\Delta f=\frac{1}{\sqrt{|g|}} \sum_{i, j}\left(\sqrt{|g|} g^{i, j} f_{j}\right)_{i}, \quad d \mu(x)=\sqrt{|g|(x)} d x,
$$

where $|g|$ is the determinant of the metric tensor $\left(g_{i, j}\right)$ and $\left(g^{i, j}\right)=\left(g_{i, j}\right)^{-1}$. Let $B(x, r)$ denote the geodesic ball of radius $r$ around $x$ and set $V(x, r)=\mu(B(x, r))$.

A smooth function $u$ is harmonic in an open set $\Omega$ if $\Delta u=0$ in $\Omega$. The heat kernel of $M$ is the minimal positive solution $(t, x, y) \mapsto p(t, x, y)$ of

$$
\left\{\begin{array}{l}
\partial_{t} u=\Delta u \text { on }(0, \infty) \times M \\
u(0, x)=\delta_{y}(x)
\end{array}\right.
$$

It is a smooth function of the three variables $(t, x, y)$ on $(0, \infty) \times M \times M$. For instance, if $M=\mathbf{R}^{n}$ equipped with its Euclidean structure,

$$
p(t, x, y)=\left(\frac{1}{4 \pi t}\right)^{n / 2} \exp \left(-\frac{\|x-y\|^{2}}{4 t}\right) .
$$

Although we will not use it explicitly in the sequel, recall that there exists a Markov process $\left(X_{t}\right)_{t \geq 0}$ having continuous paths and such that, for any bounded continuous function $f$,

$$
e^{t \Delta} f(x)=\int_{M} p(t, x, y) f(y) d \mu(y)=\mathbb{E}_{x}\left(f\left(X_{t}\right)\right) .
$$

In particular, $\int_{M} p(t, x, y) d \mu(y) \leq 1$ (in fact, for the class of manifolds of interest to us here, $\left.\int_{M} p(t, x y) d \mu(y)=1\right)$. The process $\left(X_{t}\right)_{t \geq 0}$ is called the Brownian motion on $M$. For background information, see the excellent survey [56].

1.3. Graphs. For our purpose, a graph is a pair $(X, E)$ where $E \subset X \times X$ is symmetric (i.e., $(x, y) \in E$ if and only if $(y, x) \in E$ ). The set $X$ is the vertex set and the elements of $E$ are called edges. Two vertices $x, y \in X$ are neighbors $(x \sim y)$ if and only if $(x, y) \in E$. Our graphs are oriented but symmetric so that orientation is merely a notational convention. There is a loop at $x$ if $(x, x) \in E$. We do not consider multiple edges. For $x \in X$, set

$$
N(x)=\#\{y \in X:(x, y) \in E\} .
$$

The integer $N(x)$ is the degree of the vertex $x$ (we will only consider locally finite graphs, i.e., $N<\infty$. In fact, most of our graphs will have uniformly bounded 
degree). For a subset $A \subset X$, set $N(A)=\sum_{x \in A} N(x)$. Thus the function $N$ defines the "volume" on the graph $(X, E)$. For any edge $e=(x, y)$ and any function $f$ on $X$, set $d f(e)=f(y)-f(x)$. The graph distance $d(x, y)$ between two points $x$ and $y$ in $X$ is the minimal number of edges one has to cross to move from $x$ to $y$. A graph is connected if $d(x, y)<\infty$ for all $x, y$. Set $B(x, r)=\{y: d(x, y) \leq r\}$ and $V(x, r)=N(B(x, r))$. Denote by $\mathcal{C}_{0}(X)$ the set of all finitely supported functions on $X$.

The simple random walk on $(X, E)$ is a stochastic process $\left(X_{n}\right)_{0}^{\infty}$ with values in $X$ evolving as follows. If $X_{n}=x$ then $X_{n+1}$ is one of the $N(x)$ neighbors of $x$ chosen uniformly at random. This Markov process is associated with the kernel

$$
K(x, y)=\left\{\begin{array}{cl}
1 / N(x) & \text { if } y \sim x \\
0 & \text { otherwise }
\end{array}\right.
$$

We can also view $K$ as a self-adjoint bounded operator acting on $L^{2}(X, N)$ by

$$
K f(x)=\frac{1}{N(x)} \sum_{y \sim x} f(y) .
$$

The iterated kernel $K_{n}(x, y)$ is defined inductively by

$$
K_{1}=K, \quad K_{n}(x, y)=\sum_{z} K_{n-1}(x, z) K(z, y) .
$$

In this setting, a harmonic function in a set $\Omega$ is a function $u$ defined on $\Omega_{1}=\{x$ : $d(x, \Omega) \leq 1\}$ and such that $K u=u$ in $\Omega$.

1.4. Cayley graphs. Let $\Gamma$ be a finitely generated group equipped with a finite symmetric generating set $S$. The Cayley graph $(G, S)$ is the oriented symmetric graph with vertex set $G$ and an edge from $x$ to $y$ if and only if $y=x s$ for some $s \in S$. Thus, the edge set $E$ is

$$
E=\{(x, x s): x \in \Gamma, s \in S\} .
$$

The distance $d(x, y)$ from $x$ to $y$ is the smallest $k$ such that $y=x s_{1} \ldots s_{k}$ with $s_{i} \in S, i=1, \ldots, k$. Set $B(x, r)=\{y \in \Gamma: d(x, y) \leq r\}$ and $V(x, r)=\# B(x, r)$. Obviously, these objects depend on the choice of $S$. Note that, to be consistent with the notation introduced above for general graphs, we should have set $V(x, r)=$ $|S| \# B(x, r)$ instead.

Given a probability measure $q$ on $\Gamma$, the left-invariant random walk on $\Gamma$ driven by $q$ is the discrete Markov process $\left(X_{n}\right)$ which evolves as follows. If the position at time $n$ is $X_{n}=x$ then pick $s$ in $\Gamma$ with probability $q(s)$ and move to $X_{n+1}=$ $x s$. Thus, if the process starts from $x$ at time 0 , the probability that $X_{n}=y$ is $q^{(n)}\left(x^{-1} y\right)$ where $q^{(n)}$ is the $n$-fold convolution of $q$ with itself (recall that $u *$ $v(x)=\sum_{y} u(y) v\left(y^{-1} x\right)$ ). A function $u$ is $q$-harmonic on $\Gamma$ if $u * q=u$. When $q=q_{S}=(\# S)^{-1} \mathbf{1}_{S}$, the corresponding random walk is called the simple random walk on $(G, S)$. For instance, if $\Gamma=\mathbb{Z}$ and $S=\{+1,-1\}$ then

$$
q_{S}^{n}(x)=2^{-n}\left(\begin{array}{c}
n \\
(n-x) / 2
\end{array}\right)
$$

if $n-x$ is even and $q_{S}^{(n)}(x)=0$ otherwise. In particular,

$$
q_{S}^{(2 n)}(0)=2^{-2 n}\left(\begin{array}{c}
2 n \\
n
\end{array}\right) \sim(\pi n)^{-1 / 2} .
$$


On the $d$-dimensional square lattice $\mathbb{Z}^{d}$ with $S=\left\{ \pm e_{i}, 1 \leq i \leq d\right\}$, we have

$$
q_{S}^{(2 n)}(0) \sim c_{d} n^{-d / 2}, \quad c_{d}=2(d / 4 \pi)^{d / 2} .
$$

For background on random walks, see Spitzer's book $[\mathbf{1 1 4}]$ and $[\mathbf{7 8 , 1 3 1 ]}$.

1.5. Algebraic properties. Throughout this survey, we will encounter several classes of groups defined by certain algebraic properties. For the convenience of the reader, we gather here the main definitions. See, e.g., [107] for details.

Solvable groups. A group $G$ is solvable if it admits a descending normal series $G=H_{1} \supset H_{2} \supset \cdots \supset H_{k+1}=\{e\}$ such that $H_{i} / H_{i+1}$ is abelian. It is polycyclic if there is such a series with $H_{i} / H_{i+1}$ cyclic. Polycyclic groups are always finitely generated hence countable. They are obviously solvable. One of the essential differences between polycyclic groups and general solvable groups is that subgroups of a polycyclic group are always finitely generated whereas a non-polycyclic finitely generated solvable groups always has (abelian) subgroups that are not finitely generated. In fact, polycyclic groups are exactly those solvable groups all of whose subgroups (equivalently, abelian subgroups) are finitely generated.

The lower central series of a group $G$ is the non-increasing sequence of subgroups defined by $G=G_{1}, G_{i+1}=\left[G_{i}, G\right], i=2, \ldots$ A group is nilpotent if there is a $k$ such that $G_{k+1}=\{e\}$. Finitely generated nilpotent groups are always polycyclic.

Examples of solvable groups. A typical finitely generated nilpotent group is the group $\mathrm{Up}_{n}(\mathbb{Z})$ of all $n \times n$ upper-triangular matrices with integer coefficients and diagonal entries all equal to 1 ("Up" stands for upper and for unipotent!).

The semidirect products $G=\mathbb{Z} \ltimes_{A} \mathbb{Z}^{2}, A \in S L_{2}(\mathbb{Z})$, where the product is given by

$$
(k, x)\left(k^{\prime}, x^{\prime}\right)=\left(k+k^{\prime}, x+A^{k} x^{\prime}\right)
$$

for $k, k^{\prime} \in \mathbb{Z}$ and $x, x^{\prime} \in \mathbb{Z}^{2}$ are polycyclic groups. For instance, $A=\left(\begin{array}{ll}2 & 1 \\ 1 & 1\end{array}\right)$ gives a polycyclic group having exponential volume growth. This group is isomorphic to a lattice in the three dimensional Lie group which defines the so-called "Sol" geometry in the theory of 3-manifolds [116].

Examples of solvable groups that are not polycyclic can be obtained as follows. Consider the affine group of the real line $\operatorname{Aff}(\mathbb{R})$, i.e., the group of all affine transformations $x \mapsto a x+b, a>0, b \in \mathbb{R}$, under composition. Fix a real $\lambda>1$ and consider the subgroup $\mathbb{A}_{\lambda}$ of $\operatorname{Aff}(\mathbb{R})$ generated by the transformations $x \mapsto x+1$ and $x \mapsto \lambda x$. This group is solvable but, for most values of $\lambda$, it is not polycyclic. In fact, it is polycyclic if and only if the group $T_{\lambda}$ of all translations contained in $\mathbb{A}_{\lambda}$ is finitely generated. For instance, if $\lambda=k>1$ is an integer then $T_{\lambda}$ is the ring $\mathbb{Z}[1 / k] \subset \mathbb{Q}$ which, as a group, is not finitely generated.

Wreath products. The following construction is known to play an important role in the theory of solvable groups. Let $A$ and $B$ be two finitely generated groups. Consider the algebraic direct sum $A_{B}=\sum_{b \in B} A_{b}$ of a countable number of copies of $A$ indexed by $B$. Thus $A_{B}$ is the set of all sequences $\left(a_{b}\right)_{B}$ where all but a finite number of $a_{b}$ are trivial (i.e., equal to the neutral element in $A$ ). The group law in $A_{B}$ is product coordinate by coordinate. Now, define the wreath product $A$ < $B$ as the semidirect product $A_{B} \rtimes_{\tau} B$ where the action $\tau$ is given by $\tau_{c}\left(\left(a_{b}\right)\right)=\left(a_{c^{-1} b}\right)$ for all $\left(a_{b}\right) \in A_{B}$ and $c \in B$. Thus the product in $A$ \& $B$ is given by

$$
(u, c) .(v, d)=\left(u \tau_{c}(v), c d\right), \quad u, v \in A_{B}, c, d \in B .
$$


If $A$ and $B$ are finitely generated, so is $A$ \B although it contains the subgroup $A_{B}$ which is not finitely generated unless $B$ is finite. The reader should work out this definition for $A=\{0,1\}=\mathbb{Z}_{2}$ and $B=\mathbb{Z}$. The group $\mathbb{Z}_{2} \succ \mathbb{Z}$ (also known as the lamplighter group) is an example of a solvable group which is not polycyclic. When iterating this construction, parentheses should be used with care. Moreover, iterated wreath products obtained through the above definition are distinct from the groups obtained by the more general iterating procedure described in $[\mathbf{1 0 7}]$.

Amenability. Recall that a "mean" on a discrete group $G$ is a continuous linear functional $\nu$ defined on the space of all bounded functions such that $f \geq 0 \Rightarrow$ $\nu(f) \geq 0$ and $\nu(1)=1$. A mean is invariant if for any $g \in G, \nu\left(f_{g}\right)=\nu(f)$ where $f_{g}(x)=f(g x)$. A group is amenable if it admits an invariant mean. All abelian groups are amenable and so are all solvable groups because amenability is preserved by quotient, extension, passage to a subgroup and increasing limit. Note however that the existence of a mean on $\mathbb{Z}$ requires the use of the axiom of choice. See e.g., [95, Chapter 0].

The free group $\mathbb{F}_{r}$ on $r \geq 2$ generators is an example of a group that is not amenable and so is $S L_{2}(\mathbb{Z})$. See [95, Chapter 0]. The surface group $S_{g}$, i.e., the fundamental group of a compact surface of genus $g>1$ is non-amenable. May be the most surprising non-amenable groups are the Burnside groups $B(r, n)$ (exponent $n$ and $r$ generators) for large enough odd exponent $n$ and $r \geq 2$ [1] (Adian's proof uses the co-growth criteria of Grigorchuk $[\mathbf{5 1}, \mathbf{5 3}])$.

It should also be noted that it is not always easy to decide whether a group is amenable or not. One currently popular example is Thompson's group $F$ defined by the presentation (see, e.g., [19])

$$
\left.\left\langle x_{0}, x_{1}, \ldots\right| x_{i}^{-1} x_{n} x_{i}=x_{n+1} \text { for } i<n\right\rangle .
$$

The group $F$ is generated by $x_{0}, x_{1}$. In fact, it is finitely presented and has several interesting realizations. It has exponential growth. Whether this group is amenable or not is an open problem.

1.6. Regular coverings. Let $M$ be a complete Riemannian manifold. Assume that there exists a discrete subgroup $\Gamma$ of the group of isometries of $M$ such that $N=M / \Gamma$ is a compact Riemannian manifold (we write $M / \Gamma$ even so we always think of $\Gamma$ as acting on the left). Such a manifold $M$ is called a regular covering (or cover) of $N$ with deck transformation group $\Gamma$. If we consider the fundamental groups $\pi_{1}(M), \pi_{1}(N)$ then $\pi_{1}(M)$ is a normal subgroup of $\pi_{1}(N)$ and $\Gamma \cong \pi_{1}(N) / \pi_{1}(M)$. We can realize $\Gamma$ as net in $M$ by picking an arbitrary origin $o \in M$ and considering $\Gamma o=\{\gamma o: \gamma \in \Gamma\}$. One calls this "a net" because there are positive constants $c, C$ such that any point $x \in M$ is at distance at most $C$ of $\Gamma o$ and any two points in $\Gamma o$ are at least distance $c$ apart.

By construction, the local geometry of a Riemannian co-compact regular cover is uniformly under control. More precisely, fix $r_{0}>1$. Then there are positive finite constants $c, C$ such that $c \leq \mu(B) \leq C$ for all balls of radius between $r_{0}$ and $1 / r_{0}$. Moreover, for all $x \in M$ and $0<r \leq r_{0}, V(x, r) \leq C V(x, 2 r)$. Any co-compact regular cover satisfies uniform local Harnack inequalities, both elliptic and parabolic. In particular, for all $t \geq 1 / r_{0}$ and for all $x, y, z$ with $d(y, z) \leq r_{0}$,

$$
c p(t, x, x) \leq p(t, y, z) \leq C p(t, x, x) .
$$

Of course, any co-compact regular cover has curvature bounded below and positive injectivity radius. 
1.7. Quasi-isometries. The following definition is useful to capture in a very general form the idea that a co-compact regular cover and its deck transformation group are similar. See, e.g., $[\mathbf{2 2}, \mathbf{5 8}, \mathbf{5 9}, \mathbf{7 4}]$. It owes its fame to the work and ideas of Gromov.

Definition 1.1. Let $(X, d),\left(X^{\prime}, d^{\prime}\right)$ be two metric spaces. A map $\psi: X \rightarrow X^{\prime}$ is a quasi-isometry from $X$ to $X^{\prime}$ if there are constants $C_{1}, \ldots, C_{5}$ such that:

(a) For all $x^{\prime} \in X^{\prime}$, there exists $x \in X$ such that $d^{\prime}\left(x^{\prime}, \psi(x)\right) \leq C_{1}$;

(b) For all $x, y \in X, C_{2} d(x, y)-C_{3} \leq d^{\prime}(\psi(x), \psi(y)) \leq C_{4} d(x, y)+C_{5}$.

Property (a) says that no points in $X^{\prime}$ are very far from $\psi(X)$. Property (b) says that distances are roughly preserved at large scale. If $\psi$ is as in Definition 1.1, there is a quasi-isometry $\psi^{\prime}$ from $X^{\prime}$ to $X$ such that $\sup _{x \in X} d\left(\psi^{\prime} \circ \psi(x), x\right)<\infty$.

It is not hard to check that a regular Riemannian covering $M$ of a compact manifold with deck transformation group $\Gamma$ is quasi-isometric to any fixed Cayley graph $(\Gamma, S)$. Also, any two Cayley graphs of a given finitely generated group are quasi-isometric (with the identity map as quasi-isometry). More generally, if $\left(\Gamma_{i}, S_{i}\right), i \in\{1,2\}$, are two Cayley graphs and $\Gamma_{2}$ is either a subgroup of $\Gamma_{1}$ with finite index or a quotient of $\Gamma_{1}$ by a finite subgroup then these two Cayley graphs are quasi-isometric.

Definition 1.2. Let $u, v$ be two positive functions defined on a neighborhood of $\infty$ in $\mathbb{R}_{+}$or $\mathbb{N}$. We say that $u$ dominates $v(u \gg v)$ if there are positive finite constants $a, b, c, a \leq b$, such that, for all $t$ large enough,

$$
v(t) \leq c \sup _{a t \leq s \leq b t} u(t), \quad \inf _{a t \leq s \leq b t} v(t) \leq c u(t) .
$$

We say that $u$ and $v$ are equivalent $(u \approx v)$ if $u$ dominates $v$ and vice versa.

This definition is useful to construct quasi-isometric invariants. Note that it simplifies considerably if one of the two functions $u, v$ is monotone. Indeed, if either $u$ or $v$ is monotone then $u \gg v$ is equivalent to say that there exist two positive finite constants $c, b$ such that, for all $t$ large enough, $v(t) \leq c u(b t)$.

Example 1.3. For any metric space $(X, d)$ equipped with a measure $\mu$, define the volume growth of $(X, d, \mu)$ as the $\approx$-equivalence class of $v(r)=\mu(B(o, r))$ where $o$ is an arbitrary fixed point in $X$. In general, the volume growth is not a quasiisometric invariant. However, it is preserved under quasi-isometry if we restrict our attention to spaces such that

$$
\sup _{x \in X} \frac{\mu(B(x, 2 r))}{\mu(B(x, r))}=C(r)
$$

is finite for each $r>0$ and to quasi-isometries $\psi:(X, d, \mu) \rightarrow\left(X^{\prime}, d^{\prime}, \mu^{\prime}\right)$ such that

$$
\forall x \in X, \quad c \leq \frac{\mu(B(x, 1))}{\mu^{\prime}(B(\psi(x), 1))} \leq C
$$

for some finite positive constants $c, C$.

\section{Some quasi-isometric invariants}

2.1. Bounded geometry. It will be convenient to consider the collection BG (bounded geometry) of metric measure spaces which we now describe. By definition, any space in $\mathbf{B G}$ either is a complete Riemannian manifold equipped 
with its Riemannian distance and measure or is a connected graph equipped with the graph distance and the measure $N(N(x)$ is the degree of $x)$. The manifolds in BG are exactly those with Ricci curvature bounded below and with a uniform lower bound on the volume of balls of radius 1 . The graphs in BG are those with uniformly bounded degree.

If one thinks in terms of metric measure spaces, the above definition is obviously very narrow minded but it will serve our purpose for the present survey.

It is not hard to see that any manifold in BG is quasi-isometric to some graph(s) in BG (and vice-versa). From a local viewpoint, manifolds in BG are similar to regular coverings in that their local geometry is uniformly under control. The volume function is uniformly doubling as long as the radius stays bounded above. Balls of radius 1 all have comparable volume. Uniform local Harnack inequalities, elliptic and parabolic are satisfied thanks to the groundbreaking work of S-T. Yau and his collaborators on analysis on manifolds with Ricci curvature bounded below, see, e.g., $[24,80,134]$ and $[22,111]$.

2.2. Volume growth. The following simple result goes back to $[44,113]$ and follows from the discussion in Example 1.3. Indeed, for manifolds with Ricci curvature bounded below, the Bishop-Gromov volume comparison theorem gives (see, e.g., [22, Theorem 3.10])

$$
\sup _{x \in M} V(x, 1)<\infty \text { and } \sup _{\substack{x \in M \\ 0<r \leq R}} \frac{V(x, 2 r)}{V(x, r)}<\infty .
$$

Proposition 2.1. In BG, the volume growth is a quasi-isometric invariant. In particular, a regular Riemannian covering of a compact manifold has the same volume growth as its deck transformation group.

Note in particular that the volume growth of a finitely generated group is independent of the choice of a symmetric finite generating set.

Example 2.2. The fundamental group of a compact manifold $N$ with negative sectional curvature has exponential volume growth because its volume growth is comparable to that of the universal cover of $N$ which is exponential (see, e.g., $[\mathbf{2 2}$, Theorem 3.7]).

Example 2.3. The fundamental group of a compact $n$-manifold $N$ with nonnegative Ricci curvature has volume growth $\ll r^{n}$. Indeed, if $V(x, r)$ is the volume function on the universal cover of $N$ and $V_{n}(r)=c_{n} r^{n}$ is the $n$-dimesnional Euclidean volume, Bishop's volume comparison theorem (e.g., [22, Theorem 3.9]) gives $V(x, r) \leq V_{n}(r)$.

Proposition 2.1 shows that the study of the volume growth of finitely generated groups is of great importance for the development of the large scale analysis on Riemannian co-compact covers. A good source of information on this subject is [63]. We recall the following results.

(0) Finitely generated groups have at most exponential volume growth.

(1) Non-amenable groups have exponential growth ([48]).

(2) For finitely generated discrete subgroups of almost connected Lie groups, the volume is either exponential or polynomial $r^{d}$ for some $d=0,1,2, \ldots$ $([13,61,92,117,133])$. 
(2) For finitely generated solvable groups, the volume is either exponential or polynomial $r^{d}$ for some $d=0,1,2, \ldots([\mathbf{1 3}, \mathbf{6 1}, \mathbf{9 0}, \mathbf{1 3 3}])$. This led Milnor to ask whether the growth function of any group is either exponential or polynomial. Grigorchuk surprised many by showing that the answer is no. See (4) below.

(3) A nilpotent group $H$ has polynomial volume growth $r^{d}$ with

$$
d=\sum_{1}^{k} i \operatorname{rank}\left(H_{i} / H_{i+1}\right)
$$

where $H_{1}=H, H_{i}=\left[H_{i-1}, H\right], k$ is the smallest integer such that $H_{k+1}=$ $\{e\}$, and $\operatorname{rank}(A)$ denotes the torsion free rank of the abelian group $A$. See $[\mathbf{1 3}, \mathbf{6 1}]$ and the discussion in $[\mathbf{6 3}]$.

(4) There are many finitely generated groups of intermediate growth, that is, whose volume growth is slower than exponential but is faster than polynomial. Such groups where first discovered by Grigorchuk. See [52] and the references therein.

(5) A group such that $\liminf _{r \rightarrow \infty} r^{-D} V(r)<\infty$ for some finite $D$ contains a nilpotent subgroup of finite index $([\mathbf{5 8}, \mathbf{1 1 8}])$. This is Gromov's celebrated result on groups with polynomial growth.

Specialists of volume growth conjecture that there are no groups with volume growth faster than any polynomial but slower than $\exp \left(r^{1 / 2}\right)$ (see, e.g., [63, p. $203]$ and [52]). Not only did Grigorchuk give examples of groups with intermediate volume growth, he also proved that the set of all possible growths has the cardinality of the continuum and that there are groups $G_{1}, G_{2}$ with volume growths $v_{1}, v_{2}$ such that neither $v_{1} \ll v_{2}$ nor $v_{2} \ll v_{1}$ hold true. See [52]. At this writing, there are no examples of groups of intermediate growth for which the ( $\approx$-equivalence class of) the volume growth is precisely known.

2.3. Parabolicity. On any complete Riemannian manifold $M$, set

$$
G(x, y)=\int_{0}^{\infty} p(t, x, y) d t
$$

If, for $x \neq y, G(x, y)<\infty$ then the function $G$ is called the Green function on $M$. It is the minimal positive solution of $\Delta_{y} G(x, y)=-\delta_{x}(y)$. When $G(x, y)=\infty$ for some (equivalently any) $x \neq y$, one says that $M$ is parabolic. A manifold $M$ is parabolic if and only if the Brownian motion $\left(X_{t}\right)_{t \geq 0}$ on $M$ is recurrent, i.e., almost surely, $\left(X_{t}\right)_{t \geq 0}$ visits any fixed non-empty relatively compact open set infinitely often (see, e.g., $[\mathbf{5 6}]$ ). It is proved in $[\mathbf{2 4}]$ that Riemannian manifolds with $\liminf _{r \rightarrow \infty} r^{-2} V\left(x_{0}, r\right)<\infty$ are parabolic (see the discussion in [33] for further references).

On a connected graph $(X, E)$, the simple random walk is recurrent (i.e., almost surely $\left(X_{n}\right)$ visits any fixed point infinitely often) if and only if $\sum_{n} K^{n}(x, x)=\infty$. When $\sum_{n} K^{n}(x, x)=\infty$, we say that $(X, E)$ is recurrent or, equivalently, parabolic. For a finitely generated group, we say that $\Gamma$ is recurrent (equivalently parabolic) if and only if the Cayley graph $(\Gamma, S)$ is recurrent for at least one symmetric finite generating set $S$. A walk that is not recurrent is called transient. For the following theorem, see $[\mathbf{3}, \mathbf{7 3}, \mathbf{1 1 9}, \mathbf{1 2 0}, \mathbf{1 2 5}]$. 
THEOREM 2.4. In BG, parabolicity is a quasi-isometric invariant. In particular, a co-compact regular cover is parabolic if and only if its deck transformation group is parabolic.

Sketch of The PRoOF. It turns out that parabolicity can be described in many different ways. One useful way is that a graph is non-parabolic if and only if, for some/any point $o \in X$ there exists a constant $C(o)$ such that

$$
\forall f \in \mathcal{C}_{0}(\Gamma), \quad|f(o)|^{2} \leq C(o) \sum_{E}|d f|^{2} .
$$

Similarly, a manifold is non-parabolic if and only if

$$
\forall f \in \mathcal{C}_{0}^{\infty}(M), \quad \int_{U}|f|^{2} d \mu \leq C(U) \int_{M}|\nabla f|^{2} d \mu
$$

for one/any non-empty open precompact set $U$. These criteria go back to Deny [39]. See also $[83,121]$.

Consider for instance two distinct Cayley graphs $\left(\Gamma, S_{1}\right),\left(\Gamma, S_{2}\right)$ of a given finitely generated group $\Gamma$. Then it is not hard to see that there are finite positive constants $c, C$ such that

$$
c \sum_{E_{1}}|d f|^{2} \leq \sum_{E_{2}}|d f|^{2} \leq C \sum_{E_{1}}|d f|^{2}
$$

where $E_{1}, E_{2}$ are the corresponding edge sets. It follows from the criterion above that if the simple random walk on a particular Cayley graph of a finitely generated group $\Gamma$ is recurrent then the simple random walk on any of its Cayley graphs is recurrent. Thus recurrence (i.e., parabolicity) is a property of the group $\Gamma$, i.e., it does not depend on the choice of the finite symmetric generating set $S$. Theorem 2.4 extends this to show that parabolicity is a quasi-isometric invariant in BG. To see how to pass from a manifold to a graph and vice versa, see the proof of Theorem 2.8 below.

Pòlya was the first to realize that the simple random walk on the square lattice $\mathbb{Z}^{d}$ is recurrent in dimension $d=1,2$ and transient otherwise. The question of finding exactly which finitely generated groups are recurrent emerged from the work of Kesten in the sixties and was solved around 1985 by Varopoulos. Varopoulos' proof uses Gromov's theorem on groups of polynomial volume growth. See Section 4.2 below.

TheOREM 2.5. A finitely generated group is recurrent (i.e., parabolic) if and only if it is a finite extention of $\{e\}, \mathbb{Z}$ or $\mathbb{Z}^{2}$, that is, if and only if it contains one of group $\{e\}, \mathbb{Z}$ or $\mathbb{Z}^{2}$ as a subgroup with finite index.

Corollary 2.6. Let $M$ be a regular Riemannian covering of a compact manifold and assume that $M$ is parabolic. Then $M$ covers a (possibly different) compact manifold with deck transformation group equal to either $\{e\}, \mathbb{Z}$, or $\mathbb{Z}^{2}$.

Proof. Let $M$ cover the compact manifold $N$ with deck transformation group $\Gamma$. If $M$ is parabolic, so is $\Gamma$ by Theorem 2.4. By Theorem 2.5, $\Gamma$ contains a subgroup $H$ equals to $\{e\}, \mathbb{Z}$ or $\mathbb{Z}^{2}$ and with finite index in $\Gamma$. Taking the quotient of $M$ by $H$ gives a compact Riemannian manifold covered by $M$ with deck transformation group $H$. 
Let us quote another corollary (due to G. Mess). For the definition of quasiconformal maps, we refer the reader to $[\mathbf{4 , 6 5}]$ and the references therein.

Corollary 2.7. Let $M$ be a 2-dimensional Riemannian manifold in BG. Assume that $M$ is quasi-isometric to a finitely generated group and quasi-conformally equivalent to the plane $\mathbb{R}^{2}$. Then $M$ is quasi-isometric to $\mathbb{R}^{2}$.

Sketch of THE PROOF. In dimension 2, parabolicity is a quasi-conformal invariant. Thus $M$ is parabolic. Let $\Gamma$ be the finitely generated group which is quasi-isometric to $M$. By Theorem 2.4, $\Gamma$ is parabolic. By Theorem 2.5, $\Gamma$ is a finite extention of $\{e\}, \mathbb{Z}$ or $\mathbb{Z}^{2}$. It is easy to rule out $\{e\}$ and $\mathbb{Z}$ because $M$ is quasi-conformal to the plane.

The question naturally arises of generalizing this result. For instance, the author asked several years ago the following question.

Question 1. Let $M$ be a 3 dimensional manifold in $\mathbf{B G}$ quasi-isometric to a finitely generated group and quasi-conformally equivalent to $\mathbb{R}^{3}$. Is $M$ quasiisometric to $\mathbb{R}^{3}$ ?

Recently, Maillot made interesting advances in this direction [87]. Let $M_{0}$ be a regular co-compact Riemannian covering. Say that $M_{0}$ is "large-scale conformally rigid" if, for any finitely generated group $\Gamma$ which is quasi-isometric to a manifold conformally equivalent to $M_{0}$ and of bounded geometry, $\Gamma$ is quasi-isometric to $M_{0}$ (Maillot's definition of bounded geometry is more restrictive than the one used in the definition of $\mathbf{B G}$ ). It is proved in $[\mathbf{8 7}]$ that $\mathbb{R}^{3}$ (in fact, any 3-manifold $M_{0}$ which is conformally flat and homeomorphe to $\mathbb{R}^{3}$ ) is large scale conformally rigid. This gives a positive answer to Question 1 (up to the different definitions of bounded geometry, a technicality).

Say a manifold is not $p$-parabolic if for one/any relatively compact non-empty open set $U$, there is a constant $C(U)$ such that

$$
\forall f \in \mathcal{C}_{0}^{\infty}(M), \quad \int_{U}|f|^{p} d \mu \leq C(U) \int|\nabla f|^{p} d \mu .
$$

This notion of $p$-parabolicity is equivalent to others found in the literature on quasi-regular maps and non-linear potential theory. See, e.g., [33]. By using $p$ parabolicity with $p=3$, it is easy to see that the manifold $M$ in Question 1 is quasi-isometric to $\mathbb{Z}^{d}$ with $d=0,1,2$ or 3 . See the sketch of the proof of Theorem 2.5 and $[\mathbf{1 2 8}$, Chapter X]. As before, it is not very hard to eliminate $\{e\}$ and $\mathbb{Z}$. I did not know how to eliminate $\mathbb{Z}^{2}$ but Maillot is able to use the simple connectedness at infinity of $\mathbb{R}^{3}$ to conclude that $\mathbb{Z}^{3}$ is the only possibility.

One can ask the same question in higher dimension. If a manifold is quasiisometric to a group and is $n$-parabolic then the group must be of polynomial growth of degree at most $n$. In dimension 4 , the possible groups are (up to finite extentions) $\mathbb{Z}^{d}$ with $d=0,1,2,3,4$ and the discrete Heisenberg group $\mathrm{Up}_{3}(\mathbb{Z})$.

2.4. Spectral gaps. On a Riemannian manifold $M$, for any $1 \leq p<\infty$, let $\lambda_{p}(M)$ be the largest real $\lambda$ such that

$$
\lambda \int|f|^{p} d \mu \leq \int|\nabla f|^{p} d \mu
$$


for all smooth functions $f$ with compact support. For $p=2, \lambda_{2}(M)$ is the bottom of the $L^{2}$-spectrum of $-\Delta$ and, for any $x \in M$,

$$
\lambda_{2}(M)=-\lim _{t \rightarrow \infty} t^{-1} \log p(t, x, x) .
$$

See [37, Prop.12] and the references therein. For $p=1, \lambda_{1}(M)$ is an isoperimetric constant. Indeed, by the familiar co-area formula, $(2.2)$ with $p=1$ is equivalent to

$$
\lambda \mu(A) \leq \mu^{\prime}(\partial A)
$$

for all compact sets $A$ with smooth boundary $\partial A$. Here $\mu^{\prime}$ denotes the induced Riemannian measure on the hypersurface $\partial A$.

On a graph $(X, E)$ with vertex set $X$ and (symmetric oriented) edge set $E$, let $\lambda_{p}(X)$ be the largest real $\lambda$ such that

$$
2 \lambda \sum_{X}|f|^{p} N \leq \sum_{E}|d f|^{p}
$$

When $p=2, \lambda_{2}(X)$ is the bottom of the $L^{2}$-spectrum of the operator $(I-K)$. Moreover, for any $x \in X$,

$$
1-\lambda_{2}(X)=\lim _{n \rightarrow \infty} K^{2 n}(x, x)^{1 / 2 n}
$$

If $(X, E)$ is a Cayley graph $(\Gamma, S)$ then $K(x, y)=q_{S}\left(x^{-1} y\right)$, the operator $K$ is convolution by $q_{S}$ and $K^{2 n}(x, x)=q^{(2 n)}(e)$.

On graphs, the discrete version of the co-area formula asserts that

$$
\sum_{E}|d f|=2 \int_{0}^{\infty} \# \partial\{f>t\} d t
$$

for any function $f \geq 0$ with finite support. Thus, when $p=1,(2.3)$ is equivalent to

$$
\lambda N(A) \leq \# \partial A
$$

for all finite sets $A \subset X$. Here $N(A)=\sum_{A} N$ and $\partial A$ is the boundary of $A$ defined as the set of those oriented edges $e=(x, y)$ in $E$ such that $x \in A$ and $y \in X \backslash A$.

TheOREM 2.8. In $\mathbf{B G}$, for each $p \in[1, \infty)$, the property " $\lambda_{p}>0$ " is a quasiisometric invariant. In particular, for a regular covering $M$ of a compact manifold with deck transformation group $\Gamma, \lambda_{p}(M)>0$ if and only if $\lambda_{p}(\Gamma)>0$.

Sketch of the Proof. (See, e.g., $[\mathbf{3}, \mathbf{3 6}, \mathbf{7 4}]$ ) We only discuss the case of a co-compact regular cover $M$ with deck transformation group $\Gamma$. Identify $\Gamma$ with the subset $\left\{\gamma_{o}: \gamma \in \Gamma\right\}$ of $M$ and consider a partition of unity $\chi_{\gamma}, \sum_{\Gamma} \chi_{\gamma} \equiv 1$, such that each $\chi_{\gamma}$ is a smooth compactly supported bump function centered around $\gamma o \in M$ and whose "profile" is essentially independent of $\gamma$. Then, we can consider the maps:

$$
\begin{aligned}
\text { rst } \quad: \quad \mathcal{C}_{0}^{\infty}(M) \rightarrow \mathcal{C}_{0}(\Gamma), \quad f \mapsto \operatorname{rst}(f)=\sum_{\gamma}\left(\int f \chi_{\gamma} d \mu\right) \mathbf{1}_{\gamma} \\
\text { ext } \quad: \quad \mathcal{C}_{0}(\Gamma) \rightarrow \mathcal{C}_{0}^{\infty}(M), \quad f \mapsto \operatorname{ext}(f)=\sum_{\gamma} f(\gamma) \chi_{\gamma} .
\end{aligned}
$$

These two maps are sort of "inverse" of each others. The maps rst "restricts" smooth functions defined on $M$ to functions on $\Gamma$ whereas ext "extends" functions defined on $\Gamma$ to smooth functions on $M$. Moreover, if we restrict our attention to 
non-negative functions, these two maps essentially preserve the size of the function and the size of its gradient. More precisely, for non-negative functions,

$$
\begin{gathered}
\sum_{\Gamma}|f|^{p} \leq C \int_{M}|\operatorname{ext}(f)|^{p} d \mu, \quad \int_{M}|\operatorname{ext}(f)|^{p} d \mu \leq C \sum_{\Gamma}|f|^{p} \\
\int_{M}|\nabla(\operatorname{ext}(f))|^{p} d \mu \leq C \sum_{E}|d f|^{p}
\end{gathered}
$$

and

$$
\begin{gathered}
\sum_{\Gamma}|\operatorname{rst}(f)|^{p} \leq C\left(\int_{M} f^{p} d \mu+\int_{M}|\nabla f|^{p} d \mu\right) \\
\int_{M} f^{p} d \mu \leq C\left(\sum_{\Gamma}|\operatorname{rst}(f)|^{p}+\int_{M}|\nabla f|^{p} d \mu\right), \\
\sum_{E}|d(\operatorname{rst}(f))|^{p} \leq C \int_{M}|\nabla f|^{p} d \mu .
\end{gathered}
$$

where $E=\{(\gamma, \gamma s): \gamma \in \Gamma, s \in S\}, S$ being a fixed symmetric finite generating set of $\Gamma$. As $\lambda_{p}$ can be computed by using only non-negative functions, the theorem follows. The two crucial properties of manifolds in BG used in this discretization procedure are that, for any $r>0$, (a) all balls of radius in $(r, 2 r)$ have comparable volumes and (b) all balls of radius in $(r, 2 r)$ satisfy the Poincaré inequality

$$
\int_{B}\left|f-f_{B}\right|^{p} d \mu \leq C(p, r) \int_{2 B}|\nabla f|^{p} d \mu
$$

where $f_{B}$ is the mean value of $f$ over $B$.

Proposition 2.9. In $\mathbf{B G}$, for $1 \leq p, q<\infty$, the properties " $\lambda_{p}>0$ " and " $\lambda_{q}>0$ " are equivalent.

Sketch of the Proof. (See, e.g., $[3, \mathbf{3 6}, \mathbf{4 0}, \mathbf{7 4}, \mathbf{1 2 1}]$ ) Using the change of function $f \rightarrow|f|^{s}$ for an appropriate $s>1$ and Hölder inequality, it is easy to see that $\lambda_{p}>0$ implies $\lambda_{q}>0$ for all $q \geq p \geq 1$. For instance, on a Riemannian manifold, for non-negative functions and $s>1$, we have $\left|\nabla f^{s}\right|=s f^{s-1}|\nabla f|$. Hence, if $q>p, s=q / p$ and $s^{\prime}=s /(s-1)$ then

$$
\begin{aligned}
\int_{M}\left|\nabla f^{s}\right|^{p} d \mu & =s^{p} \int_{M} f^{(s-1) p}|\nabla f|^{p} d \mu \\
& \leq s^{p}\left(\int f^{(s-1) p s^{\prime}} d \mu\right)^{1 / s^{\prime}}\left(\int_{M}|\nabla f|^{p s} d \mu\right)^{1 / s} \\
& \leq s^{p}\left(\int f^{q} d \mu\right)^{1-1 / s}\left(\int_{M}|\nabla f|^{q} d \mu\right)^{1 / s} .
\end{aligned}
$$

It follows that $\lambda_{q} \geq(p / q)^{q} \lambda_{p}^{q / p}$. Up to technical details, the same proof works on graphs.

To prove the converse, we will work on graphs and appeal to Theorem 2.8 to conclude in the case of manifolds (the proof given for graphs does not easily adapt to manifolds). Let $(X, E)$ be a graph with $\lambda_{p}(X)>0$. According to what we just proved, it suffices to show that $\lambda_{1}(X)>0$. Setting $f=\mathbf{1}_{A}$ in $(2.3)$ we obtain

$$
\lambda_{p}(X) N(A) \leq \# \partial A
$$


for any finite set $A \subset X$ is a finite set. By the discrete co-area formula, (2.4) is equivalent to

$$
2 \lambda_{p}(X) \sum|f| N \leq \sum_{E}|d f| .
$$

This shows that $\lambda_{p}(X) \leq \lambda_{1}(X)$ as desired. It follows from this discussion that, for graphs, $\lambda_{p}>0 \Leftrightarrow \lambda_{q}>0$ for all $1 \leq q, p<\infty$. By Theorem 2.8, this is also true in BG.

Now we recall the following fundamental results of Følner [48] and Kesten $[\mathbf{7 6}, \mathbf{7 7}]$. Without using the present terminology, Følner was working essentially with the isoperimetric constant $\lambda_{1}$. Kesten was interested in random walks and the spectral constant $\lambda_{2}$. At that time (1955-59), the relation between the two was not clearly established.

THEOREM 2.10. A finitely generated group $\Gamma$ satisfies $\lambda_{p}>0$ for one/any $p \in[1, \infty)$ if and only if it is non-amenable.

Together, the two theorems above give the following well-known statement due to Brooks $[\mathbf{1 8}]$ (see also $[\mathbf{3}, \mathbf{1 1 9}]$; a different proof is in $[\mathbf{1 1 2}]$ ).

THEOREM 2.11. The covering $M$ of a compact manifold satisfies $\lambda_{p}(M)>0$ for one/any $p \in[1, \infty)$ if and only if its deck transformation group is non-amenable.

Example 2.12. Let $S_{g}$ be the fundamental group of a compact surface of genus $g>1$. Then $S_{g}$ is quasi-isometric to the hyperbolic plane. Hence $\lambda_{p}\left(S_{g}\right)>0$ for all $p \in[1, \infty)$ and thus $S_{g}$ is non-amenable. More generally, the fundamental group of a compact manifold admitting a metric of negative sectional curvature is non-amenable.

2.5. Isoperimetry. Let $\Psi$ be an arbitrary positive function. We say that a graph $(X, E)$ satisfies the $\Psi$-isoperimetric inequality if

$$
N(A) \leq \Psi(N(A)) \# \partial A .
$$

If we set

$$
I(t)=\inf \{\# \partial A: A \subset X, N(A)=t\}
$$

then $t / I(t)$ is the best possible $\Psi$ for the graph $(X, E)$. These definitions should be handled with care because the so-called isoperimetric profile $I$ is not an increasing function in general. Even for Cayley graphs, it is not known if $I$ is $\approx$-equivalent to the monotone isoperimetric profile $I^{\uparrow}(k)=\inf \{\# \partial A: A \subset X, N(A) \geq k\}$. Moreover, $I^{\uparrow}$ is inappropriate for the discussion of inequalities of the form (2.5). Indeed, the smallest non-decreasing $\Psi$ satisfying (2.5) is

$$
J(k)=\sup \left\{\frac{N(A)}{\# \partial A}: N(A) \leq k\right\} .
$$

Even for Cayley graphs, it is not known if $J(k) \approx k / I(k)$ nor if $J(k) \approx k / I^{\uparrow}(k)$.

The Følner function of $(G, S)$ is defined by setting

$$
F(t)=\min \left\{N(A): \text { there exists } A \subset X \text { such that } \# \partial A<t^{-1} N(A)\right\} .
$$

This is a non-decreasing function. It is related to $J$ by

$$
F(t)>k \Leftrightarrow J(k) \leq t
$$

The following is an elementary result. 
Proposition 2.13. For graphs in $\mathbf{B G}, I, I^{\uparrow}, J, F$ are all $\approx$-invariant under quasi-isometry.

On a complete Riemannian manifold $M$, we define the isoperimetric functions $I, I^{\uparrow}, J$ of $M$ and the Følner function $F$ of $M$ by replacing $N(A)$ by $\mu(A)$ and \# $\partial A$ by $\mu^{\prime}(\partial A)$, working with precompact sets $A$ with smooth boundary instead of finite sets. In particular, we say that $M$ satisfies the $\Psi$-isoperimetric inequality if

$$
\mu(A) \leq \Psi(\mu(A)) \mu^{\prime}(\partial A)
$$

for all relatively compact open sets $A$ with smooth boundary.

It is not entirely obvious how to distinguish between small scale and large scale isoperimetry on a manifold (see [60, Lemma 6.21] and $[\mathbf{2 8 , 2 3}]$ ). To state a precise and fairly general result, we will use Young conjugate functions and Orlicz norms (for a detailled treatment of Orlicz spaces, see [75]; for their use in the present context, see, e.g., $[\mathbf{9}, \mathbf{7 0}, \mathbf{8 9}])$. Let $P, Q$ be a pair of conjugate Young functions (this implies that $P, Q$ are convexe increasing functions). Define the Orlicz $P$-norm of a function $f$ by

$$
\|f\|_{P}=\sup \left\{\int f g d \mu: \int Q(|g|) d \mu \leq 1\right\} .
$$

The Orlicz $P$-norm of the caracteristic function of a set $U$ is

$$
\left\|\mathbf{1}_{U}\right\|_{P}=\mu(U) Q^{-1}(1 / \mu(U))
$$

where $Q^{-1}$ is the inverse function of $Q$. It follows from the co-area formula that the isoperimetric inequality

$$
\mu(A) \leq \Psi(\mu(A)) \mu^{\prime}(\partial A)
$$

for relatively compact sets with smooth boundary is equivalent to the Sobolev inequality (See $[\mathbf{8 9}]$ )

$$
\forall f \in \mathcal{C}_{0}^{\infty}(M), \quad\|f\|_{P} \leq\|\nabla f\|_{1}
$$

as long as $\Psi$ and $P$ are related by $\Psi(t)=1 / Q^{-1}(1 / t)$. As $1 / Q^{-1}(1 / t)$ is increasing we can state this equivalence as

$$
\forall f \in \mathcal{C}_{0}^{\infty}(M), \quad\|f\|_{P} \leq\|\nabla f\|_{1} \Leftrightarrow J(t) \leq 1 / Q^{-1}(1 / t) .
$$

Proposition 2.14. Let $P, Q$ be a pair of Young conjugate functions and assume that $P$ satisfies $P(2 t) \leq C P(t)$ for all $t>0$. Set $\Psi(t)=1 / Q^{-1}(1 / t)$. Let $M_{1}, M_{2} \in$ BG (thus each $M_{i}$ is either a graph or a manifold with bounded geometry). Assume that $M_{1}$ and $M_{2}$ satisfy the local isoperimetric inequality

$$
\mu(A) \leq C \Psi(\mu(A))\left[\mu(A)+\mu^{\prime}(\partial A)\right]
$$

and that $M_{1}$ and $M_{2}$ are quasi-isometric. Then $M_{1}$ satisfies $J(t) \ll \Psi(t)$, that is,

$$
\mu(A) \leq C \Psi(\mu(A)) \mu^{\prime}(\partial A)
$$

if and only if $M_{2}$ does.

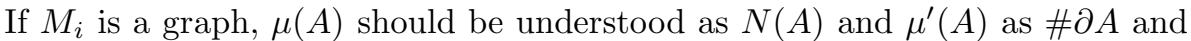
in this case the hypothesis that $M_{i}$ satisfies $N(A) \leq C \Psi(\mu(A))[N(A)+\# \partial A]$ is trivially satisfied because $\psi$ is increasing and $N(A)$ is bounded away form 0 . It is also useful to note that the fact that $P$ is doubling implies that $P^{-1}$ and $Q^{-1}$ (hence $\Psi$ ) are also doubling although $Q$ may not be doubling. 
Sketch of the Proof of Proposition 2.14. The proof of Proposition 2.14 is along the same lines as the proof of Theorem 2.8. If $M_{1}$ and $M_{2}$ are both graphs, Proposition 2.13 gives a more precise result. Thus its suffices to treat the case where $M_{1}=X$ is a graph and $M_{2}=M$ is a manifold. For simplicity, we outline the proof in the case when $M$ is a regular covering of a compact manifold with deck transformation group $\Gamma$. We keep the notation introduced in the sketch of the proof of Theorem 2.8. In addition to the maps rst and ext introduced in that proof, we consider the regularization map reg : $\mathcal{C}_{0}^{\infty}(M) \mapsto \mathcal{C}_{0}^{\infty}(M)$ defined by

$$
\operatorname{reg}(f)(x)=\sum_{\gamma}\left(\frac{\int f \chi_{\gamma} d \mu}{\int \chi_{\gamma} d \mu}\right) \chi_{\gamma} .
$$

It is plain that $\|\operatorname{reg}(f)\|_{P} \approx\|\operatorname{rst}(f)\|_{P}$. It is easy to show that a $\psi$-isoperimetric inequality on $M$ implies the same type of inequality on $\Gamma$ (more generaly, $X$ ). To go from $\Gamma$ to $M$, write

$$
\|f\|_{P} \leq\|\operatorname{reg}(f)\|_{P}+\|f-\operatorname{reg}(f)\|_{P} .
$$

Observe that $\|\operatorname{reg}(f)\|_{P} \approx\|\operatorname{rst}(f)\|_{P}$ and estimate this term using the $P$-Sobolev inequality on $\Gamma$ and $\sum_{E}|d(\operatorname{rst}(f))| \leq C \int_{M}|\nabla f| d \mu$. To estimate the second term $\|f-\operatorname{reg}(f)\|_{P}$, use the local $P$-Sobolev inequality $\|f\|_{P} \leq C\left[\|f\|_{1}+\|\nabla f\|_{1}\right]$ (which is equivalent to (2.7)) and the inequality $\|f-\operatorname{reg}(f)\|_{1} \leq C\|\nabla f\|_{1}$.

Let $V(t)$ be the volume growth function of a finitely generated group $\Gamma$ (for some finite symmetric set of generators) and set $W(t)=\inf \{s>0: V(s)>t\}$. It is proved in [35] that $\Gamma$ satisfies the isoperimetric inequality $J(t) \ll W(t)$, that is

$$
\# A \leq C W(C \# A) \# \partial A .
$$

For instance, if $V(t) \gg t^{d}$ then $J(t) \ll t^{1 / d}$ whereas $V(t) \gg \exp \left(t^{\alpha}\right)$ implies $J(t) \leq[\log (1+t)]^{1 / \alpha}$. Comments are in order concerning (2.8) since in his wonderful and very influencial book [60, Chap. 6, E+], Gromov gives a very misleading and erroneous account of the discovery of this inequality. Varopoulos was the first to obtain valuable general isoperimetric inequalities on groups based on volume growth. He proved (2.8) for groups of polynomial growth $[\mathbf{1 2 2}, \mathbf{1 2 3}, \mathbf{1 2 4}]$ using several structure theorems such as Malcev's embedding of any torsion free finitely generated nilpotent group as a co-compact lattice in a nilpotent Lie group and Gromov's theorem on groups of polynomial volume growth. He also proved that groups of super-polynomial growth satisfy $J(t) \ll t^{1 / d}$ for all $d>0$ and that $J(t) \ll[\log (1+t)]^{2 / \alpha}$ if $V(t) \gg \exp \left(t^{\alpha}\right)$ (see $\left.[\mathbf{1 2 3}, \mathbf{1 2 6}, \mathbf{1 2 7}]\right)$. In [126], Varopoulos conjectured that $V(t) \gg \exp \left(t^{\alpha}\right) \Longrightarrow J(t) \ll[\log (1+t)]^{1 / \alpha}$ (as noted above, this follows immediately from (2.8)). The method used by Varopoulos to prove the partial results mentioned above are not suited to handle general volume growth functions and, as far as I know, they cannot be used to prove (2.8) or even the much simpler implication $V(t) \gg \exp \left(t^{\alpha}\right) \Longrightarrow J(t) \ll[\log (1+t)]^{1 / \alpha}$. The proof of (2.8) in [35] uses a variation on an idea used by D. W. Robinson in [106] to prove a Nash inequality on Lie groups.

By Proposition 2.14, we obtain the following result (see [35, Théorème 4] and $[96,97,128])$.

TheOREM 2.15. Let $M$ be a n-dimensional Riemannian regular covering of a compact manifold. Let $V(o, r)$ be the volume growth function from a fixed origin o. 
- If $V(o, r) \gg r^{d}$ for some $d>0$ then $M$ satisfies the $\Psi$-isoperimetric inequality (2.6) where $\Psi(t)$ is a concave function satisfying $\Psi(t) \approx t^{1 / n}$ at $t=0$ and $\Psi(t) \approx t^{1 / d}$ at $t=\infty$.

- If $V(o, r) \gg \exp \left(r^{\alpha}\right)$ with $\alpha \in(0,1]$ then $M$ satisfies the $\Psi$-isoperimetric inequality (2.6) where $\Psi(t)$ is a concave function satisfying $\Psi(t) \approx t^{1 / n}$ at $t=0$ and $\Psi(t) \approx(\log t)^{1 / \alpha}$ at $t=\infty$.

- If the deck transformation group of $M$ contains a nilpotent group $H$ of finite index then

$$
J(t) \approx \begin{cases}t^{1 / n} & \text { at } 0 \\ t^{1 / d} & \text { at } \infty\end{cases}
$$

where $d$ is given by (2.1) applied to the group $H$.

- If the deck transformation group of $M$ contains a polycyclic group $H$ of finite index and exponential volume growth then

$$
J(t) \approx \begin{cases}t^{1 / n} & \text { at } 0 \\ \log t & \text { at } \infty .\end{cases}
$$

- If the deck transformation group of $M$ is not amenable then

$$
J(t) \approx\left\{\begin{array}{cl}
t^{1 / n} & \text { at } 0 \\
1 & \text { at } \infty .
\end{array}\right.
$$

For refined results concerning the very special case of periodic metrics on $\mathbb{R}^{n}$, see $[\mathbf{9 4}]$.

The results of $[\mathbf{4 5}, \mathbf{9 9}]$ and Proposition 2.14 give examples of regular coverings of compact manifolds whose volume growth is exponential and whose isoperimetric profile $J$ at infinity is intermediate between the extreme case $J(t) \approx 1$ (non-amenable) and $J(t) \approx \log t$ (polycyclic of exponential growth). The simplest example is the wreath product $\mathbb{Z} \imath \mathbb{Z}$ which has $J(t) \approx(\log t) /(\log \log t)$. See [45] where there are also examples for which $J(t)$ behaves at infinity as $(\log t)^{1 / k}$ with $k$ an integer, or $\left(\log _{m} t\right)^{1 / k}$ where $m, k$ are integers and $\log _{m}$ denotes the $m$-iterated logarithm.

Question 2 ([103]). Let $\Gamma$ be a solvable group with exponential volume growth and finite Prüfer rank (i.e., there exists $k$ such that any finitely generated subgroup of $\Gamma$ contains a generating set with atmost $k$ elements). Prove that $J(t) \approx \log t$.

Pittet and the author make the following conjecture.

CONJECTURE 1. For any finitely generated torsion free solvable group with exponential growth, $J(t) \approx \log t$ if and only if the group has finite Prüfer rank.

The group $\mathbb{Z}_{2} \prec \mathbb{Z}$ is solvable with exponential growth. It is not of finite Prüfer rank and satisfies $J(t) \approx \log t$ but it is not torsion free.

\section{Invariance of the heat decay}

3.1. Questions concerning the invariance of the heat decay in BG. On a manifold, define

$$
\phi_{M}(x, t)=p(t, x, x), \quad \phi_{M}(t)=\sup _{x \in M} p(t, x, x) .
$$

On a graph set

$$
\phi_{X}(x, n)=K_{2 n}(x, x), \quad \phi_{X}(n)=\sup _{x \in X} K_{2 n}(x, x) .
$$


In what follows we will often write $M$ for either a graph or a manifold. In great generality, thanks to the local parabolic Harnack inequality, we have

$$
0<c(x, y) \leq \lim _{t \rightarrow \infty} \frac{\phi_{M}(x, t)}{\phi_{M}(y, t)} \leq C(x, y)<\infty .
$$

Thus $\phi_{M}(x, t) \approx \phi_{M}(y, t)$ for any pair $x, y$. Note that it may easily happen that the $\phi_{M}(x, t)$ is not $\approx$ equivalent to $\phi_{M}(t)$. For instance, one may have $\phi_{M}(x, t) \approx t^{-d / 2}$ whereas $\phi_{M}(t) \approx t^{-D / 2}$ with $0<d<D<\infty$ arbitrary integers. See, e.g., [37, 57].

In view of the results presented in the previous sections, one may ask the following question.

QUeStion 3. In $\mathbf{B G}$, is the $\approx$-equivalence class of the functions $\phi_{M}(x, \cdot)$, $x \in M$, a quasi-isometric invariant? Is the $\approx$-equivalence class of the function $\phi_{M}$ a quasi-isometric invariant?

A more precise form of these questions is as follows.

QUESTION 4. Let $M_{1}, M_{2}$ be two objects in $\mathbf{B G}$ (thus, each is either a manifold or a graph). Let $\psi$ be a quasi-isometry from $M_{1}$ to $M_{2}$. Are there constants $0<$ $c \leq 1 \leq C<\infty$ such that, for all $x \in M$ and all $t \geq 1$,

$$
c \phi_{M_{1}}(x, C t) \leq \phi_{M_{2}}(\psi(x), t) \leq C \phi_{M_{1}}(x, c t) ?
$$

3.2. Invariance for Cayley graphs and regular covers. For a Cayley graph $(\Gamma, S)$, we have

$$
\phi_{\Gamma}(x, n)=\phi_{\Gamma}(n)=q_{S}^{(2 n)}(e) .
$$

For a cover of a compact manifolds, there is a positive finite constant $c$ such that

$$
\forall x \in M, c \phi_{M}(t) \leq \phi_{M}(x, t) \leq \phi_{M}(t) .
$$

Thus, in these cases, we consider only the functions $\phi_{\Gamma}(t), \phi_{M}(t)$. We call "heat decay" of $\Gamma$ (resp. $M)$ the $\approx$-equivalence class of $\phi_{\Gamma}\left(\operatorname{resp} . \phi_{M}\right)$. The following result is taken from $[\mathbf{1 0 0}]$.

TheOREM 3.1. For Cayley graphs and regular covers of compact manifolds the heat decay is a quasi-isometric invariant. In particular, a regular cover of a compact manifold and its deck transformation group share the same heat decay.

In more concrete terms, if $M$ covers a compact manifold with deck transformation group $\Gamma$, and if $S$ is any fixed symmetric finite set generating $\Gamma$, there are positive finite constants $c, C$ such that

$$
\forall x \in M, \forall n \in\{1,2, \ldots\}, \quad c p(C n, x, x) \leq q_{S}^{(2 n)}(e) \leq C p(c n, x, x) .
$$

What is perhaps remarkable about this result is that, in the present generality, we know very little about the behavior of $p(n, x, x) \approx q^{(2 n)}(e)$. In the next section we review what is known about $q^{(2 n)}(e)=\phi_{\Gamma}(n)$ under various algebraic assumptions on $\Gamma$.

\section{The heat decay for random walks}

This section reviews what is known about the heat decay for finitely generated groups. Of course, by Theorem 3.1, most of the results described below can be translated in the context of regular covers of compact manifolds.

Fix a finitely generated group $\Gamma$. We assume that $\Gamma$ is equipped with a finite symmetric generating set $S$. Distance and volume growth on $\Gamma$ are computed 
with respect to $S$. Theorem 3.1 shows that the $\approx$-equivalence class of $\phi_{(\Gamma, S)}$ is independent of the choice of $S$. By definition, it is the heat decay of $\Gamma$ and we denote it by $\phi_{\Gamma}$.

4.1. Further stability results. We start by collecting some results from [100] that complement Theorem 3.1.

THEOREM 4.1. Let $\Gamma$ be a finitely generated group. Let $\Gamma^{\prime}$ be a finitely generated subgroup of a quotient of $\Gamma$. Then $\phi_{\Gamma} \ll \phi_{\Gamma^{\prime}}$.

Note that this applies in particular to quotients and to subgroups of $\Gamma$.

TheOREM 4.2. Let $\Gamma$ be a finitely generated group. Let $q$ be a symmetric probability measure on $\Gamma$.

- Assume that $\sum_{\Gamma}|\gamma|^{2} q(\gamma)<\infty$. Then $\phi_{\Gamma} \ll \phi_{q}$.

- Assume that $q$ has finite generating support. Then $\phi_{q} \ll \phi_{\Gamma}$.

It may be surprising at first that such a wide variety of probability measures shares the same basic behavior. However, in $\mathbb{Z}^{d}$, it is well-known that the second moment condition $\sum_{\gamma}|\gamma|^{2} q(\gamma)<\infty$ delimits the natural domain of application of the central limit theorem.

4.2. From volume growth to the heat decay. Suppose that $M$ is a graph or manifold in BG and that we are given a uniform lower bound

$$
\forall x, \forall r>1, \quad V(x, r) \geq v(r)
$$

where $v$ is a continuous positive increasing convex function. What can be said about the heat decay? The answer found in [12] is that $\phi_{M}$ is then bounded above by the inverse function of $s v^{-1}(s)$ where $v^{-1}$ is the inverse function of $v$. Hence, if $v(r)=c r^{d}$ with $d \geq 1$ then $\phi_{M}(t) \leq C t^{d /(1+d)}$. If $v(r)=\exp (c r)$ then $\phi_{M}(t) \leq C t / \log t$. Moreover, examples show that these results are essentially sharp.

For Cayley graphs, the situation is much better thanks to the following result. The precise form given here is from $[\mathbf{2 7}, \mathbf{1 0 4}]$. The two special cases emphasized in the statement below were obtained earlier in ground breaking works by Varopoulos. See $[\mathbf{1 2 7}, \mathbf{1 2 8}]$.

THEOREM 4.3. Let $\Gamma$ be a finitely generated group with finite symmetric generating set $S$. Let $w(t)=\inf \{n: V(n)>t\}$. Define $\psi$ implicitely for $t$ large enough by

Then $\phi_{\Gamma} \ll \rho$. In particular:

$$
t=\int_{1}^{1 / \rho(t)} \frac{w(s)^{2} d s}{s} .
$$

- if $V(n) \gg n^{d}$ then $\phi_{\Gamma}(t) \ll t^{-d / 2}$.

- if $V(n) \gg \exp \left(n^{\alpha}\right)$ then $\phi_{\Gamma}(t) \ll \exp \left(-t^{\alpha /(\alpha+2)}\right)$.

The proof of this remarkable result although not very difficult is too long to be included here. The crucial ingredient that catches the difference between, say, general graphs of bounded degree and Cayley graphs is the following "Calculustype" inequality. For any $f \in \mathcal{C}_{0}(\Gamma)$, we have

$$
\forall y \in \Gamma, \quad \sum_{x \in \Gamma}|f(x y)-f(x)| \leq|y|_{S} \sum_{E}|d f| .
$$

The proof of this inequality is the only place where the group structure is used in the proof of Theorem 4.3. 
Example 4.4. Let $\Gamma$ be a finitely generated group. Assume that $\Gamma$ contains a infinite normal subgroup $Z_{1}$, the quotient $\Gamma / Z_{1}$ contains an infinite normal subgroup $Z_{2}$ and so on, $k$ times. Then $\Gamma$ has volume growth $V(n) \gg n^{k}$ (this simple result is noted in $[\mathbf{5 8}, \mathrm{p} .59])$. Thus $\phi_{\Gamma}(n) \ll n^{-k / 2}$.

Theorem 4.3 is one of the keys to the proof of Theorem 2.5 (i.e., to Varopoulos' solution of Kesten's conjecture concerning recurrent groups). Indeed, Theorem 4.3 shows that a recurrent group must satisfy $\liminf r^{-\alpha} V_{S}(r)<\infty$ for each $\alpha>2$ (if not, we would have $V(r) \gg r^{\alpha}$ for some $\alpha>2$. By Theorem 4.3, this would give $\phi_{\Gamma}(n) \ll n^{-\alpha / 2}$ and thus $\phi_{\Gamma}(n)$ would be summable, contradicting the hypothesis that $\Gamma$ is recurrent). By Gromov's theorem on groups of polynomial volume growth, this implies that $V_{S}(n) \approx n^{d}$ with $d=0,1$ or 2 and it is not hard to show that the only groups with such growth are the finite extensions of $\{e\}, \mathbb{Z}$ and $\mathbb{Z}^{2}$.

Coulhon and Grigor'yan [31] give a simple and general proof that if $V_{S}(n) \approx r^{d}$ then $\phi_{\Gamma}(n) \gg n^{-d / 2}$ (properly stated, their result is not restricted to groups). Alexopoulos $[2]$ shows that polycyclic groups with exponential volume growth satisfy $\phi_{\Gamma}(n) \gg \exp \left(-n^{1 / 3}\right)$. Thus the results stated in Theorem 4.3 are sharp on some examples.

4.3. Classical behavior and discrete linear groups. There are several equivalent definitions of polycyclic groups (see Section 1.5). In particular, polycyclic groups are exactly those countable solvable groups that can be realized as a closed subgroup of a Lie group with finitely many connected components. See [105]. Moreover, it turns out that any discrete subgroup of a connected Lie group is either non-amenable or contains a polycyclic subgroup of finite index. This follows from the work of Tits [117] and Mostow [92]. Thus, for discrete subgroups of connected Lie groups, we have the following (see, e.g., [98]).

THEOREM 4.5. Let $\Gamma$ be a discrete subgroup of a real Lie group with finitely many connected components. Then either ( $a$ ) $\Gamma$ is non-amenable or ( $b$ ) $\Gamma$ is finitely generated and contains a polycyclic subgroup of finite index. In the second case, either $\Gamma$ has exponential volume growth and satisfies $\phi_{\Gamma}(n) \approx \exp \left(-n^{1 / 3}\right)$ or $\Gamma$ has polynomial volume growth $V(r) \approx r^{d}$ and satisfies $\phi_{\Gamma}(n) \approx n^{-d / 2}$ for some $d=0,1,2, \ldots$

Thus for a finitely generated discrete subgroup $\Gamma$ of a connected Lie group there are only three cases: $\phi_{\Gamma} \approx \exp (-n), \phi_{\Gamma} \approx \exp \left(-n^{1 / 3}\right), \phi_{\Gamma} \approx n^{-d / 2}$ for some integer $d$. We call these three behaviors the classical behaviors.

4.4. Exotic behaviors for solvable groups. Because of the great variety of groups of intermediate growth discovered by Grigorchuk [52], it seems unlikely that one can obtain a complete classification of the possible behaviors of $\phi_{\Gamma}$ in full generality. One might, however, dream of a classification of all possible behaviors of $\phi_{\Gamma}$ for finitely generated solvable groups. Indeed, this would be very easy is $\phi_{\Gamma}$ was determined by the volume growth for solvable groups but it is not as the following examples show.

Example 4.6 $([\mathbf{9 9}, \mathbf{1 0 1}, \mathbf{1 0 2}])$. Let $\lambda$ be a real that is not algebraic. Then the subgroup of $\mathbb{A}_{\lambda}$ of $\operatorname{Aff}(\mathbb{R})$ generated by $x \mapsto x+1$ and $x \mapsto \lambda x$ is isomorphic to the wreath product $\mathbb{Z} \imath \mathbb{Z}$ and satisfies $\phi_{\mathbb{A}_{\lambda}}(n) \approx \exp \left(-n^{1 / 3}(\log n)^{2 / 3}\right)$. 
Not only is $\mathbb{Z} \imath \mathbb{Z}$ solvable, it is in fact metabelian and even abelian by cyclic. It is proved in [102] that, for any abelian by cyclic group $\Gamma$ with exponential growth, either $\phi_{\Gamma}(n) \approx \exp \left(-n^{1 / 3}\right)$ or $\mathbb{Z} \imath \mathbb{Z} \subset \Gamma$ and $\phi_{\Gamma}(n) \approx \exp \left(-n^{1 / 3}(\log n)^{2 / 3}\right)$. Thus, the volume growth is not sufficient to determine the behavior of $\phi_{\Gamma}$ in the class of solvable groups. This fact is more dramatically illustrated by the following examples, all of exponential volume growth (each of the different classes of groups considered below contains solvable groups).

Example $4.7([45,97, \mathbf{1 0 1}, \mathbf{1 0 4}])$. Let $A$ and $B$ be two finitely generated groups and consider the wreath product $\Gamma=A$ భ $B$.

- Assume that $B$ has polynomial growth of degree $d$ (i.e., $\left.V(r) \approx r^{d}\right)$. Then $\phi_{\Gamma}(n) \approx\left\{\begin{array}{l}\exp \left(-n^{d /(d+2)}\right) \text { if } A \text { is finite and non trivial } \\ \exp \left(-n^{d /(d+2)}(\log n)^{2 /(d+2)}\right) \text { if } A \text { is infinite with polynomial growth } \\ \exp \left(-n^{(d+1) /(d+3)}\right) \text { if } A \text { is polycyclic with exponential growth. }\end{array}\right.$

- Assume that $B$ is polycyclic with exponential volume growth. Then for any non-trivial $A$, finite or polycyclic, we have

$$
\phi_{A \curlywedge B}(n) \approx \exp \left(-n(\log n)^{-2}\right) .
$$

- Assume that $\Gamma=A_{k} \prec\left(A_{k-1} \prec\left(\cdots\left(A_{1} \prec B\right) \cdots\right)\right)$ where each $A_{i}$ is non-trivial polycyclic. Set $\log _{k}(t)=\log \left(1+\log _{k-1}(t)\right)$ with $\log _{0}(t)=t, t>1$.

- If $B$ is polycyclic with exponential growth then

$$
\phi_{\Gamma}(n) \approx \exp \left(-n\left(\log _{k} n\right)^{-2}\right) .
$$

- If $k \geq 2$ and $B$ has polynomial growth of degree $d \geq 1$, let $V_{1}$ be the volume growth function of $A_{1}$. Then

$$
\phi_{\Gamma}(n) \approx \begin{cases}\exp \left(-n\left(\log _{k-1} n\right)^{-2 /(d+1)}\right. & \text { if } V_{1} \text { is exponential } \\ \exp \left(-n\left[\left(\log _{k-1} n\right) / \log _{k} n\right]^{-2 / d}\right) & \text { if } V_{1} \text { is polynomial, } V_{1} \not 1 \\ \exp \left(-n\left(\log _{k-1} n\right)^{-2 / d}\right) & \text { if } V_{1} \approx 1 \text {, i.e., } A_{1} \text { is finite }\end{cases}
$$

More examples can be constructed from the results in $[\mathbf{4 5}, \mathbf{1 0 1}]$. In particular, Erschler's results from [45] represent a breakthrough and provide a variety of examples. See also [47].

As mentioned above, for abelian by cyclic groups of exponential growth, only two behaviors of $\phi_{\Gamma}$ are possible. The papers $[\mathbf{1 0 1}, \mathbf{1 0 3}]$ give two other results in this direction. Recall that a group $\Gamma$ has finite Prüfer rank if there exists an integer $k$ such that any finitely generated subgroup of $\Gamma$ can be generated by $k$ elements or less.

TheOREM 4.8. Let $\Gamma$ be a finitely generated solvable group.

- If $\Gamma$ is metabelian then $\phi_{\Gamma}(n) \gg \exp \left(-n^{1-\epsilon}\right)$ for some $\epsilon \in(0,1)$.

- If $\Gamma$ has finite Prüfer rank then $\phi_{\Gamma}(n) \gg \exp \left(-n^{1 / 3}\right)$.

Pittet and the author make the following conjecture.

Conjecture 2. Let $\Gamma$ be a finitely generated solvable torsion free group. Then $\phi_{\Gamma}(n) \gg \exp \left(-n^{1 / 3}\right)$ if and only if $\Gamma$ has finite Prüfer rank.

The group $\mathbb{Z}_{2} \succ \mathbb{Z}$ is solvable with exponential growth. It is not of finite Prüfer rank and satisfies $\phi_{\mathbb{Z} Z \mathbb{Z}}(n) \approx \exp \left(-n^{1 / 3}\right)$ but it is not torsion free.

QUESTION 5. Is it true that if $\Gamma$ is metabelian of exponential growth then there exist two rational numbers $\alpha, \beta$, such that $\phi_{\Gamma}(n) \approx \exp \left(-n^{\alpha}(\log n)^{\beta}\right)$ ? 
Question 6. Let $\mathbb{S}_{d}^{2}=\mathbb{F}_{d} / \mathbb{F}_{d}^{\prime \prime}$ be the free metabelian group with d generators $\left(\mathbb{F}_{d}^{\prime \prime}\right.$ is the second derived group). What is the behavior of $\phi_{\mathbb{S}_{d}^{2}}$ ?

The study of the group $\mathbb{S}_{d}^{2}$ is advocated in [130] where the Poisson boundary is studied (see also [46]). In preliminary work, Pittet and the author observed that

$$
\exp \left(-n^{d /(d+2)}\right) \ll \phi_{\mathbb{S}_{d}^{2}}(n) \ll \exp \left(-n^{(d-1) /(d+1)}(\log n)^{2 /(d+1)}\right) .
$$

Question 7. Is it true that for any step-three solvable group $\Gamma$ there exists $\epsilon>0$ such that

$$
\phi_{\Gamma}(n) \gg \exp \left(-n(\log n)^{-\epsilon}\right) ?
$$

4.5. Isoperimetry and the heat decay on groups. It is well understood (e.g., $[\mathbf{3 0}, \mathbf{5 5}, \mathbf{1 0 4}, \mathbf{1 2 8}]$ and the different approach of $[\mathbf{9 1}])$ that, in a very general setting, control of the isoperimetric profile yields control on the uniform heat kernel decay. In general, something is lost in this type of argument (see [34]).

In the case of a Cayley graph $(\Gamma, S)$, one has the following precise statement, versions of which hold in much greater generality. Define $J_{\Gamma}=J$ by

$$
J(k)=\sup \left\{\frac{\# A}{\# \partial A}: \# A \leq k\right\}
$$

and define the function $\rho=\rho_{J}$ implicitely by

$$
t=\int_{1}^{1 / \rho(t)} J(s)^{2} \frac{d s}{s} .
$$

Theorem 4.9. The heat decay $\phi_{\Gamma}$ of the Cayley graph $(\Gamma, S)$ is bounded by

$$
\phi_{\Gamma} \ll \rho .
$$

It is also possible to estimate $J$ in terms the heat decay but the known results in this direction are less satisfactory and the following question is open.

Question 8. Let $\left(\Gamma_{i}, S_{i}\right)$ be two Cayley graphs.

- Suppose $J_{\Gamma_{1}} \approx J_{\Gamma_{2}}$. Does it implies $\phi_{\Gamma_{1}} \approx \phi_{\Gamma_{2}}$ ?

- Suppose $\phi_{\Gamma_{1}} \approx \phi_{\Gamma_{2}}$. Does it implies $J_{\Gamma_{1}} \approx J_{\Gamma_{2}}$ ?

That is, does $J$ and $\phi$ contain the same information about the underlying group?

For further discussions and results concerning this question, see $[\mathbf{3 1}, \mathbf{3 2}, \mathbf{1 0 4}]$.

4.6. The heat decay and Sobolev inequality on regular covers. This section gather statements concerning the on-diagonal behavior of the heat kernel on co-compact Riemannian covers. These statements are simple consequences of the results reviewed earlier. First, as a consequence of Theorems 4.5 and 3.1, we have the following result.

THEOREM 4.10. Let $M$ be a co-compact Riemannian regular cover. Assume that the deck transformation group of $M$ can be realized as a discrete subgroup of a real Lie group with finitely many connected components. Then the heat decay $\phi_{M}(t), t \gg 1$, is given by one of the following three exclusive possibilities:

1. $\phi_{M}(t) \approx e^{-t}$;

2. $\phi_{M}(t) \approx e^{-t^{1 / 3}}$

3. There exists an integer $d$ such that $\phi_{M}(t) \approx t^{-d / 2}$. 
Of course, the first case corresponds exactly to the case where the deck transformation group $\Gamma$ is non-amenable, the second case to the case where $\Gamma$ is amenable with exponential growth and the third case to the case where $\Gamma$ has polynomial volume growth of degree $d$. The examples of Section 4.4 show that many other behaviors are possible when $\Gamma$ cannot be realized as a discrete subgroup of a real Lie group with finitely many connected components. In such cases, the following corollary to Theorems 3.1, 4.3 may be useful.

THEOREM 4.11. Let $M$ be a co-compact Riemannian regular cover.

- Assume that $V(x, r) \geq c r^{d}, r>1$. Then, for all $t>1, \phi_{M}(t) \leq C t^{-d / 2}$.

- Assume that $V(x, r) \geq c \exp \left(c r^{\alpha}\right), r>1$. Then

$$
\forall t>1, \quad \phi_{M}(t) \leq C_{1} \exp \left(-c_{1} t^{\alpha /(2+\alpha)}\right) .
$$

The next statement connects these heat kernel estimates to the most classical form of the Sobolev inequality. See, e.g., [128].

TheOREM 4.12. Let $M$ be a co-compact Riemannian regular cover of dimension $n$. Then $M$ satisfies the Sobolev inequality

$$
\forall f \in \mathcal{C}_{0}(M), \quad\left(\int_{M}|f|^{2 \nu /(\nu-2)} d \mu\right)^{(\nu-2) / 2 \nu} \leq C \int_{M}|\nabla f|^{2} d \mu
$$

if and only if $\nu \geq n$ and $V(x, r) \geq c r^{\nu}, r>1$.

Note that the last volume condition above is satisfied if and only if the deck transformation group has volume growth at least $r^{\nu}$. One can prove other versions of the above inequality (see, e.g., $[\mathbf{1 1 0}, \mathbf{1 1 1}, \mathbf{1 2 8}]$ ). More precisely, assume that $V(x, r) \geq c r^{\nu}$ for all $r>0$ (hence, $\nu>n$ ) and fix $1 \leq p<\nu$. Then there exists a constant $C$ such that

$$
\forall f \in \mathcal{C}_{0}^{\infty}(M), \quad\|f\|_{p \nu /(\nu-p)} \leq C\|\nabla f\|_{p} .
$$

Assume in addition that the volume growth is subexponential, i.e., $\lim _{n \rightarrow \infty} V(n)^{1 / n}<$ 1. Then there exists a constant $C$ such that for any smooth function $f$ on $M$ satisfying $|\nabla f| \in L^{p}$ (not necessarily compactly supported), there exists a constant $C(f)$ for which

$$
\forall f \in \mathcal{C}_{0}^{\infty}(M), \quad\|f-C(f)\|_{p \nu /(\nu-p)} \leq C\|\nabla f\|_{p} .
$$

In cases where $\Gamma$ has polynomial volume growth or degree $d$ with $d<n$, no Sobolev inequalities of the form stated above can possibly hold but there are several ways to cope with this situation. Assume indeed that $\Gamma$ has polynomial volume growth or degree $d$ and set $\nu=\max \{n, d\}$. Then the families of local Sobolev inequalities

$$
\forall B=B(x, r), \quad \forall f \in \mathcal{C}_{0}^{\infty}(B), \quad\|f\|_{B, p \nu / \nu-p)} \leq C_{p} r \mu(B)^{-1 / \nu}\|\nabla f\|_{B, p}
$$

and

$$
\forall B=B(x, r), \quad \forall f \in \mathcal{C}^{\infty}(B), \quad\left\|f-f_{B}\right\|_{B, p \nu /(\nu-p)} \leq C_{p} r \mu(B)^{-1 / \nu}\|\nabla f\|_{B, p},
$$

hold for all $1 \leq p<\nu$ (see, e.g., [111]). Here, $\|\cdot\|_{B, p}$ is the $L^{p}$ norm restricted to the set $B$ and $f_{B}$ denotes the mean of $f$ over $B$. Alternatively, one can use the Faber-Krahn type inequality (see [55])

$$
\forall B=B(x, r), \quad \forall \Omega \subset B, \quad \lambda(\Omega) \geq \frac{c}{r^{2}}\left(\frac{\mu(\Omega)}{\mu(B)}\right)^{2 / \nu}
$$


where $\lambda(\Omega)$ denotes the lowest eigenvalue of minus the Laplacian with Dirichlet boundary condition in the open set $\Omega$.

\section{Harmonic functions}

5.1. Liouville properties. A complete Riemannian manifold is Liouville if any bounded harmonic function is constant. It has the strong Liouville property if any positive harmonic function is constant. The same definitions apply to graphs. A measure $q$ on a group is Liouville (resp. strong Liouvillle) if any bounded (resp. positive) solution of $u * q=u$ is constant.

The study of Liouville's properties is really only the iceberg's top of the deeper problem of studying the space of all bounded harmonic functions or the cone of all positive harmonic functions through the construction and understanding of the Poisson and Martin boundaries. The Liouville property is equivalent to the fact that the Poisson boundary is reduced to a singleton. In the class BG, the strong Liouville property is equivalent to the fact that the Martin boundary is reduced to a singleton. We will not discuss Poisson and Martin boundaries here. Pointers to the literature can be found in $[3,71,72,85,129,131]$.

Blackwell [16] appears to be the first to have observed that any bounded harmonic functions on the square lattice $\mathbb{Z}^{d}$ must be constant. This is a special case of the celebrated Choquet-Deny theorem [25] which implies that, under mild conditions on the probability measure $q$, bounded $q$-harmonic functions on a locally compact abelian group are constants. Dynkin and Maljutov [43] observed that finitely generated nilpotent groups have the Liouville property. Later, the following three satisfactory results concerning the Liouville property were obtained:

- If $q$ is a probability measure with finite generating support on a group $\Gamma$ and $\Gamma$ has subexponential volume growth (i.e., $\lim _{n \rightarrow \infty} V(n)^{1 / n}=1$ ) then any bounded $q$-harmonic function is constant. See [5].

- If $q$ is a symmetric finitely supported measure on a polycyclic group then any bounded $q$-harmonic function is constant. See $[\mathbf{2}, \mathbf{6 8}]$.

- If the support of $q$ generates $\Gamma$ and $\Gamma$ is non-amenable then there are nontrivial bounded $q$-harmonic functions on $\Gamma$. See $[\mathbf{6}]$ and also [50].

Concerning the strong Liouville property, Margulis [88] proved that if $q$ is symmetric and $\Gamma$ is nilpotent then any positive $q$-harmonic function is constant. More recently, Bougerol and Elie [17] proved the following theorem and corollary (their results are slightly more general than stated here).

THEOREM 5.1. Let $\Gamma$ be a finitely generated group. Assume that there exists a continuous homomorphism $h$ from $\Gamma$ into a group $G$ having finitely many connected components such that the closure of $h(\Gamma)$ has exponential volume growth. Assume that $q$ is symmetric with generating support and has a third moment (i.e., $\left.\sum|\gamma|{ }^{3} q(\gamma)<\infty\right)$. Then there are non-constant positive $q$-harmonic functions on $\Gamma$.

COROLlary 5.2. If $q$ is symmetric, with finite generating support and $\Gamma$ is polycyclic then the positive q-harmonic functions are constant if and only if $\Gamma$ has polynomial volume growth.

Example 5.3. Theorem 5.1 applies to the subgroups $\mathbb{A}_{\lambda}$ of the affine group of the line (recall that $\mathbb{A}_{\lambda}$ is the group generated by $x \mapsto x+1$ and $x \mapsto \lambda x$ ). For any 
$\lambda>1$, and for any symmetric probability measure $q$ with finite generating support on $\mathbb{A}_{\lambda}$, there are non-constant positive $q$-harmonic functions.

5.2. Instability of the Liouville properties. In [84], Lyons gives examples of quasi-isometric pairs $M_{1}, M_{2}$ in $\mathbf{B G}$ such that $M_{1}$ admits non-constant bounded harmonic functions whereas $M_{2}$ has the strong Liouville property. He provides examples where both $M_{1}$ and $M_{2}$ are graphs, or both manifolds, or one is a graph and the other a manifold. Thus, each of the Liouville properties is unstable under quasi-isometry in BG. See also [14]. However, these works leave open the following basic questions.

Question 9. Let $\left(\Gamma_{1}, S_{1}\right)$ and $\left(\Gamma_{2}, S_{2}\right)$ be two quasi-isometric Cayley graphs. Is it true that $\left(\Gamma_{1}, S_{1}\right)$ has the (strong) Liouville property if and only if $\left(\Gamma_{2}, S_{2}\right)$ does?

QUESTION 10. Given two symmetric probability measure $q_{1}, q_{2}$ with finite generating supports on a group $\Gamma$, does the (strong) Liouville property for $q_{1}$ implies the same property for $q_{2}$ ?

QUESTION 11. Let $M$ be a regular cocompact cover with deck transformation group $\Gamma$. Let $g_{1}, g_{2}$ be two $\Gamma$ invariant Riemannian metrics on $M$. Is it true that $\left(M, g_{1}\right)$ has the (strong) Liouville property if and only if $\left(M, g_{2}\right)$ does?

5.3. Passage from the deck transformation group to the cover. Despite the fact that we do not know if the Liouville properties are stable when passing from a covering to its deck transformation group (assuming the quotient is compact), there is a way to transport some information thanks to a discretization procedure introduced by Furstenberg [49] and refined successively by Lyons and Sullivan [86], Ancona [3], Kaimanovich [69], and Ballman and Ledrappier [10] (the result in $[\mathbf{1 0}]$ is more precise than the one stated below).

Proposition 5.4. Let $M$ be a regular Riemannian covering of a compact manifold with deck transformation group $\Gamma$. There exists a symmetric probability measure $q$ on $\Gamma$ whose support is $\Gamma$, which satisfies $\sum_{\gamma \in \Gamma} e^{c|\gamma|} q(\gamma)<\infty$ for some $c>0$, and such that:

- Any bounded harmonic function on $M$ restricted to $\Gamma$ is q-harmonic.

- any positive q-harmonic function on $\Gamma$ can be extended to a positive harmonic function on $M$.

Suppose we want to apply this proposition to show that a certain co-compact Riemannian covering has (does not have) the Liouville property by using information concerning the deck transformation group $\Gamma$. Then it is not enough to have information about a specific random walk on $\Gamma$, nor does it suffice to know about all random walks driven by finitely symmetric supported measures. In the form stated above, Proposition 5.4 requires dealing with symmetric measures $\mu$ having an exponential moment. This is in contrast with what happens when a property is stable under quasi-isometries.

Example 5.5. Let $M$ be a regular co-compact cover with non-amenable deck transformation group. Then, by the aforementioned result of Azencott (any nonamenable group admits non trivial bounded harmonic functions for (essentially) any random walk) and Theorem 5.4, $M$ admits non-constant bounded harmonic function. 
Example 5.6. Let $M$ be a regular covering of a compact manifold with deck transformation group $\Gamma=\mathbb{A}_{\lambda}, \lambda>1$. (this group is isomorphic to $\mathbb{Z} \imath \mathbb{Z}$ when $\lambda$ is not algebraic). Then, using Proposition 5.4 and Theorem 5.1, we obtain that $M$ admits non-constant positive harmonic functions.

Example 5.7. Let $M$ be a regular covering of a compact manifold with deck transformation group $\Gamma$ having sub-exponential growth (i.e., $\lim V(n)^{1 / n}<1$ ). Then any bounded harmonic function on $M$ is constant. See $[\mathbf{6 7}]$ and $[\mathbf{3}$, Théorème $3.1]$.

Example 5.8. Consider the wreath products $\Gamma_{k}=\mathbb{Z}_{2} \succ \mathbb{Z}^{k}$. Then the Cayley graphs of $\Gamma_{k}$ have the Liouville property if and only if $k \leq 2[\mathbf{7 1}, \mathbf{7 2}]$. From Proposition 5.4 and [71, Theorem 3.3], it follows that a co-compact regular cover with deck transformation group $\Gamma_{k}$ has the Liouville property if and only if $k \leq 2$.

Further examples are dicussed in [46] where, in particular, a compact Riemannian manifold with amenable fundamental group whose universal cover does not have the Liouville property is constructed.

The following result is from $[\mathbf{1 7}]$. See also $[\mathbf{3}, \mathbf{8}, \mathbf{6 2}, \mathbf{6 9}]$.

TheOREM 5.9. Assume that $M$ is a regular co-compact cover whose deck transfomation group is a closed subgroup of a group having finitely many connected components. Then $M$ has non-trivial positive harmonic functions if and only if $\Gamma$ has exponential volume growth. It has non-trivial bounded harmonic functions if and only if $\Gamma$ is non-amenable.

5.4. Harmonic functions with finite energy. On a manifold, consider the energy form $D(f)=\int_{M}|\nabla f|^{2} d \mu$ whereas, on a graph, set $D(f)=\sum_{e \in E}|d f(e)|^{2}$. Royden work [108] sparkled off interest on whether or not harmonic functions with finite energy are constant. Say that the "finite energy Liouville property" holds if any harmonic function $u$ with $D(u)<\infty$ is constant.

The following result of Holopainen and Soardi [66] may be a little surprising in view of the instability of the Liouville and strong Liouville properties.

THEOREM 5.10. The finite energy Liouville property is invariant under quasiisometries in BG. In particular, a co-compact Riemannain cover has the finite energy Liouville property if and only if its deck transformation group does.

There are several simple but not completely obvious facts concerning harmonic functions with finite eneregy that are worth noting (for graphs, a good reference is $[\mathbf{1 1 5}])$. Any harmonic function of finite energy is the difference of two positive harmonic functions. Thus, if the strong Liouville property holds, so does the finite energy Liouville property. In fact, the finite energy Liouville property holds already if bounded harmonic functions with finite energy are constant. In particular, it holds if the Liouville property holds (see, e.g., [66] which treats $p$-harmonic functions).

Example 5.11. Let $M$ be a regular Riemannian co-compact cover with deck transformation group $\Gamma$. Assume that $\Gamma$ as subexponential volume growth. Then $M$ has the finite energy Liouville property.

5.5. Harnack inequalities. Assume that $M$ is a metric space equipped with a family of harmonic functions (for our purpose, it suffices to think of the examples above where $M$ is a Riemannian manifold or a Cayley graph). We say that $M$ 
satisfies the elliptic Harnack inequality if there is a constant $C$ such that for any ball $B$ and any positive function $u$ harmonic in $2 B$, we have

$$
\sup _{B}\{u\} \leq C \inf _{B}\{u\} .
$$

Let us emphasize that $C$ is independent of the ball $B$ and of the function $u$. In particular, this is in an obvious sense a scale invariant property.

The Harnack inequality implies the strong Liouville property (the converse is false as will become clear from some of the examples discussed below). Classical harmonic functions in Euclidean space satisfy the Harnack inequality. For manifolds with non-negative Ricci curvature, (5.1) was obtained in [24] under the stronger form $|\nabla u| \leq \frac{C}{r} u$ in $B$.

QUESTION 12. Is the elliptic Harnack inequality (5.1) a quasi-isometric invariant in $\mathbf{B G}$ ?

For simplicity, we define the parabolic Harnack inequality only on manifolds (see, e.g., $[\mathbf{2 9}, \mathbf{3 8}]$ for the graph version). Given a real $s, r \in(0, \infty)$ and $x \in M$, set $Q=\left(s, s+4 r^{2}\right) \times B(x, 2 r)$ and

$$
Q_{-}=\left(s+r^{2}, s+2 r^{2}\right) \times B(x, r), \quad Q_{+}=\left(s+3 r^{2}, s+4 r^{2}\right) \times B(x, r) .
$$

Thus, $Q_{-}$and $Q_{+}$are two sub-cylinders contained in $Q$ with $Q_{+}$sitting well above $Q_{-}$and $Q_{-}$floating in the bottom part of $Q$.

We say that a manifold $M$ satisfies the parabolic Harnack inequality if there exists a constant $C$ such that for any non-negative solution $u$ of $\left(\partial_{t}+\Delta\right) u=0$ in $Q$ we have

$$
\sup _{Q_{-}}\{u\} \leq C \inf _{Q_{+}}\{u\}
$$

It is proved in [36] that the parabolic Harnack inequality (5.2) is stable under quasiisometries in $\mathbf{B G}$. This is because it can be characterized by the volume doubling property and the scale invariant Poincaré inequality. See $[54,109,111]$.

5.6. A necessary condition for Harnack inequality in BG. The following useful result is due to Barlow [11].

Proposition 5.12. Let $M$ be a graph or a manifold in BG. If $M$ satisfies the Harnack inequality (5.1) then the volume growth of $M$ is bounded above by a power function.

Sketch of The PROOF. In [11], Barlow proves this result (in a more general form) for graphs. Here, we adapt Barlow's proof to manifolds. In $M$, consider a maximal set of point $X=\left\{x_{i}, i=1,2, \ldots\right\}$ such that $d\left(x_{i}, x_{j}\right)>16$ if $i \neq j$. Obviously, the (closed) ball $B\left(x_{i}, 8\right)$ are pairwise disjoint whereas the (closed) balls $B\left(x_{i}, 16\right)$ cover $M$. Fix $o \in M$. Let $F_{n}=\left\{i: B\left(x_{i}, 1\right) \cap B(o, n) \backslash B(o, n-1) \neq \emptyset\right\}$. We claim that $B(o, n)$ is covered by the balls $B\left(x_{i}, 64\right), i \in F_{n}$. Indeed, let $z$ be in $B(o, n)$. Let $j$ be such that $z \in B\left(x_{j}, 16\right)$. Along a shortest path from $x_{j}$ to $o$, let $y$ be the first point such that $d(o, y) \leq n-16$ and let $j_{1}$ be such that $y \in B\left(x_{j_{1}}, 16\right)$. Then $B\left(x_{j_{1}}, 1\right)$ intersects $B(o, n)$. As $d\left(o, x_{j}\right) \leq n+16$, we must have $d\left(x_{j}, y\right) \leq 32$ hence $d\left(x_{j}, x_{j_{1}}\right) \leq 48$. Thus $d\left(x_{j_{1}}, z\right) \leq 64$. As balls of a fixed radius all have comparable volume (because $M \in \mathbf{B G}$ ), there exists a constant $C$

$$
\mu(B(o, n)) \leq \sum_{k=0}^{n} \sum_{i \in F_{k}} \mu\left(B\left(x_{i}, 64\right)\right) \leq C \sum_{0}^{n} \# F_{k} .
$$


We now claim that there exist $C, \alpha>0$ such that

$$
\# F_{k} \leq C k^{\alpha} \text {. }
$$

If this is the case then $\mu(B(o, n)) \leq C^{\prime} n^{\alpha+1}$ which is the desired result. To prove this claim, we use the Brownian motion $\left(X_{t}\right)_{t>0}$ on $M$. Fix $k$ and set

$$
\begin{aligned}
& A=A_{k}=\cup_{i \in F_{k}} B\left(x_{i}, 1\right), \\
& \tau=\inf \left\{t>0: X_{t} \in A_{k}\right\}
\end{aligned}
$$

and

$$
h_{i}(x)=\mathbb{P}_{x}\left(X_{\tau} \in B\left(x_{i}, 1\right)\right) .
$$

Thus $h_{i}(x)$ is the probability that, starting from $x$, one first enters $A$ by entering $B\left(x_{i}, 1\right)$. By the strong Markov property, each $h_{i}$ is harmonic on $M \backslash A$.

ASSERTION 5.13. There exists a positive constant $c_{0}>0$ such that, for every $k, i \in F_{k}$, and $x$ such that $d\left(x, x_{i}\right)=2$, we have $h_{i}(x) \geq c_{0}$.

Proof. This assertion is satisfied because $M$ has bounded geometry (i.e., $M \in$ BG). To see this, for $x \in B\left(x_{i}, 8\right)$, observe that

$$
h_{i}(x) \geq u_{i}(x)=\mathbb{P}_{x}\left(X_{\tau_{i}} \in B\left(x_{i}, 1\right) \text { and } \tau_{i}<\sigma_{i}\right)
$$

where $\tau_{i}$ is the first hitting time of $B\left(x_{i}, 1\right)$ and $\sigma_{i}$ is the first exit time from $B\left(x_{i}, 8\right)$. Then, for $x \in B\left(x_{i}, 8\right)$, we have

$$
u_{i}(x) \approx \frac{g_{i}\left(x_{i}, x\right)}{g_{i}\left(x_{i}, y\right)}
$$

where $g_{i}$ is the Green function with Dirichlet boundary condition in $B\left(x_{i}, 8\right)$ and $y$ is an arbitrary point on $\partial B\left(x_{i}, 1\right)$. By well-known Dirichlet heat kernel estimates, for $z$ such that $1 \leq d\left(x_{i}, z\right) \leq 7$, we have $g_{i}\left(x_{i}, z\right) \approx 1$.

ASSERTION 5.14. There are finite positive constants $c, \alpha$ such that, for every $k$ and every $i \in F_{k}, h_{i}(o) \geq c k^{-\alpha}$.

Proof. Fix $i \in F_{k}$. Let $\gamma_{t}, 0 \leq t \leq T$ be a shortest curve from $x_{i}$ to $o$ parametrized by the distance from $x_{i}$ so that $d\left(x_{i}, \gamma_{t}\right)=t, 0 \leq t \leq T$. We need to control de distance from $\gamma_{t}$ to $A$. By construction, if $t \leq 8, d\left(\gamma_{t}, A\right)=$ $d\left(\gamma_{t}, B\left(x_{i}, 1\right)\right)=t-1$. Moreover, the triangle inequality shows that for any $j \in F_{k}$ with $j \neq i$, we have

$$
d\left(\gamma_{t}, B\left(x_{j}, 1\right)\right) \geq d\left(o, B\left(x_{j}, 1\right)\right)-d\left(o, \gamma_{t}\right) \geq t-3 .
$$

Thus, for $t \in[2, T]$,

$$
d\left(\gamma_{t}, A\right) \geq t / 2 .
$$

Now, consider the points $z_{j}=\gamma_{t_{j}}$ with $t_{j}=2(1+1 / 8)^{j-1}, j=1,2, \ldots, j_{0}$ where $j_{0}$ is such that $d\left(o, x_{j_{0}}\right) \leq k / 4$. It follows that $j_{0} \approx \log k$. For each $j=1, \ldots, j_{0}-1$, $d\left(x_{j}, x_{j+1}\right)=t_{j} / 8$ and the function $h_{i}$ is harmonic in $B\left(x_{j}, t_{j} / 2\right)$. Thus the Harnack inequality (5.1) gives $h_{i}\left(z_{j+1}\right) \geq C^{-1} h_{i}\left(z_{j}\right)$ ( $C$ being the constant from (5.1)). It follows that

$$
h_{i}\left(z_{j_{0}}\right) \geq C^{-j_{0}} h_{i}\left(z_{1}\right) .
$$

Applying (5.1) one more time to pass from $z_{j_{0}}$ to $o$ and using Assertion 5.13 to estimate $h_{i}\left(z_{1}\right)$, we obtain $h_{i}(o) \geq c_{0} C^{-j_{0}} \geq c k^{-\alpha}$ for some $c, \alpha>0$, as desired. 
To finish the proof of Proposition 5.12, we show that Assertion 5.14 implies (5.3). Indeed, as the balls $B\left(x_{i}, 1\right), i \in F_{k}$, form a partition of $A_{k}, \sum_{i \in F_{k}} h_{i}(x)=1$. Hence

$$
1 \geq \sum_{i \in F_{k}} h_{i}(o) \geq c \# F_{k} k^{-\alpha}
$$

which gives (5.3).

5.7. Analysis on regular covers having polynomial volume growth. Using Proposition 5.12, Gromov's theorem on groups of polynomial growth, and the results of $[\mathbf{5 4}, \mathbf{1 0 9}]$, we can describe those co-compact regular covers satisfying the elliptic (and parabolic) Harnack inequality.

THEOREM 5.15. Let $M$ be a Riemannian co-compact regular cover with deck transformation group $\Gamma$. The following properties are equivalent.

- The elliptic Harnack inequality (5.1) is satisfied.

- The parabolic Harnack inequality (5.2) is satisfied.

- The group $\Gamma$ has polynomial volume growth.

- The heat kernel $p(t, x, y)$ satisfies the two-sided estimate

$$
\frac{c_{1}}{V(x, \sqrt{t})} e^{-c_{2} d(x, y)^{2} / t} \leq p(t, x, y) \leq \frac{c_{3}}{V(x, \sqrt{t})} e^{-c_{4} d(x, y)^{2} / t}
$$

for all $t>0$ and $x, y \in M$.

If $M$ is a co-compact Riemannian regular cover and its deck transformation group $\Gamma$ has polynomial growth then $M$ satisfies the Poincaré inequality

$$
\forall f \in \mathcal{C}^{\infty}(B), \quad \int_{B}\left|f-f_{B}\right|^{p} d \mu \leq C_{p} r(B)^{p} \int_{B}|\nabla f|^{p} d \mu
$$

for each $p \in[1, \infty)$. Here $r(B)$ denotes the radius of that ball, $f_{B}$ is the mean of $f$ over the ball $B$, and the constant $C_{p}$ is independent of $B$ (see, e.g., [36]). Note that, by the results of $[\mathbf{5 4}, \mathbf{1 0 9}, \mathbf{1 1 1}]$, the two sided Gaussian heat kernel inequality of Theorem 5.15 holds on a co-compact cover $M$ having polynomial volume growth as soon as the Riemannian metric is uniformly comparable to a metric lifted from the compact quotient. This type of global heat kernel estimate is similar to the estimates obtained by $\mathrm{Li}$ and Yau [80] for manifolds with nonnegative Ricci curvature. Note however that, in general, the manifolds in Theorem 5.15 do not have non-negative Ricci curvature. For instance, $\Gamma$ may have volume growth $r^{d}$ with $d$ strictly larger than the dimension of $M$, preventing the existence of a Riemannian metric of non-negative Ricci curvature on $M$.

As a corollary of Theorem 5.15, we see that a regular co-compact Riemannian cover $M$ with deck transformation group of polynomial volume growth of degree $d \geq 3$ admits a Green function $G(x, y)$ which satisfies

$$
c d(x, y)^{2-d} \leq G(x, y) \leq C d(x, y)^{2-d}
$$

for all $x, y \in M$ with $d(x, y) \geq 1$. For the case of abelian cover, [7] gives the following more precise result (obtained by Fourier transform techniques).

TheOREM 5.16. Let $M$ be a regular Riemannian co-compact cover with deck transformation group equal to $\mathbb{Z}^{d}, d \geq 3$. Then there exists an Euclidean norm $\|\cdot\|$ on $\mathbb{R}^{d}$ such that the green function $G$ satisfies

$$
\lim _{\gamma \rightarrow \infty}\|\gamma\|^{d-2} G(x, \gamma(y))=c_{d}>0
$$


for all $x, y \in M$ (in the limit, $\gamma$ is an element of $\Gamma=\mathbb{Z}^{d}$ and $\gamma(y)$ is the image of $y$ under the action of $\gamma$ ).

A result that is similar in spirit to the above large scale asymptotic of the Green function and concerns the heat kernel is obtained in [82].

In a recent work, N. Dungey $[\mathbf{4 2}]$ has obtained gradient estimates that complement the results of Theorem 5.15.

THEOREM 5.17. Let $M$ be a co-compact Riemannian cover with deck transformation group $\Gamma$ of polynomial volume growth. Let $S$ be a symmetric finite generating set of $\Gamma$. Let $p(t, x, y)$ be the heat kernel on $M_{0}$. Then, for all $t>0$, and $x, y \in M_{0}$,

$$
\left|\nabla_{y} p(t, x, y)\right| \leq \frac{C}{t^{1 / 2} V(x, \sqrt{t})} \exp \left(-c d(x, y)^{2} / t\right) .
$$

Dungey uses (5.5) and further estimates obtained in [42] to prove that the Riesz transforms are bounded on $L^{p}(M, \mu), 1<p<\infty$, that is,

$$
\forall f \in \mathcal{C}_{0}^{\infty}(M), \quad c_{p}\|\nabla f\|_{p} \leq\left\|(-\Delta)^{1 / 2} f\right\|_{p} \leq C_{p}\|\nabla f\|_{p}
$$

Again, Dungey's gradient estimate is similar to the global gradient estimates that follows from [80] in the case of manifolds with non-negative Ricci curvature.

Another result worth mentioning here concerns harmonic functions of at most polynomial growth. See $[\mathbf{2 6}, \mathbf{7 9}]$.

TheOREM 5.18. Let $M$ be a co-compact Riemannian cover with deck transformation group $\Gamma$ of polynomial volume growth of degree $d$. Then there exists a constant $C$ such that the space of all harmonic functions on $M$ satisfying

$$
\sup _{r>1} \sup _{d(o, x) \leq r}\left\{r^{-\alpha}|u(x)|\right\}<\infty
$$

has finite dimension bounded by $C \alpha^{d-1}$.

An interesting and non-trivial generalization of Theorem 5.15 concerns quotients of regular covering by subgroups that are not necessarily normal. See [64, 109].

THEOREM 5.19. Let $M$ be a Riemannian co-compact regular cover with deck transformation group $\Gamma$. Assume that $\Gamma$ has polynomial volume growth. Let $\Gamma_{0}$ be a subgroup of $\Gamma$ (not necessarily a normal subgroup) and let $M_{0}=M / \Gamma_{0}$ be the Riemannian manifold obtained by taking the quotient of $M$ by $\Gamma_{0}$. Then $M_{0}$ satisfies the elliptic and parabolic Harnack inequalities (5.1), (5.2) and the heat kernel $p(t, x, y)$ on $M_{0}$ satisfies the two-sided estimate

$$
\frac{c_{1}}{V(x, \sqrt{t})} e^{-c_{2} d(x, y)^{2} / t} \leq p(t, x, y) \leq \frac{c_{3}}{V(x, \sqrt{t})} e^{-c_{4} d(x, y)^{2} / t}
$$

for all $t>0$ and $x, y \in M_{0}$.

Note that in the setting of this theorem the volume function $V(x, r)$ will often exhibit a non-uniform behavior as $x$ varies. The manifolds in Theorem 5.19 satisfy the Poincaré inequality (5.4). Dungey's gradient estimate (5.5) and the boundedness of the Riesz transforms also hold in this context. 


\section{References}

[1] S.I. Adian Random walks on free periodic groups. Math. USSR Izvestiya 21 (1983), 425-434.

[2] G. Alexopoulos A lower estimate for central probabilities on polycyclic groups. Canadian Math. J., 44 (1992), 897-910.

[3] A. Ancona Théorie du potentiel sur les graphes et les variétés. Lecture Notes in Math. 1427 (1990), Springer.

[4] G. Anderson, M. Vamanamurthy and M. Vuorinen Conformal invariants, inequalities, and quasiconformal maps. Wiley \& Sons, 1997.

[5] A. Avez Harmonic functions on groups. In "Differential Geometry and Relativity" (1976), 27-32. Reidel, Dordrecht.

[6] R. Azencott Espace de Poisson des groupes localement compacts. Lecture Notes in Math. 148 (1970), Springer.

[7] M. Babillot Théorie du renouvelement pour des chaines semi-markoviennes transientes. Ann. Inst. H. Poincaré, Probab. Stat. 24 (1988), 507-569.

[8] M. Babillot, P. Bougerol and L. Elie On the strong Liouville property for co-compact Riemannain covers. Rend. Sem. Mat. Fis. Milano 64 (1994), 77-84.

[9] D. Bakry, T. Coulhon, M. Ledoux and L. Saloff-Coste Sobolev inequalities in disguise. Indiana Univ. Math. J. 44 (1995), 1033-1074.

[10] W. Ballman and F. Ledrappier Discretization of positive harmonic functions on Riemannian manifolds and Martin boundaries. In "Actes de la table ronde en l'honneur de Marcel Berger", Séminaires et Congrés 1, Soc. Math. France, (1996).

[11] M. Barlow Some remarks on the elliptic Harnack inequality for graphs. Preprint, 2003.

[12] M. Barlow, T. Coulhon and A. Grigor'yan Manifolds and graphs with slow heat kernel decay. Inventiones Math. 144 (2001), 609-649.

[13] H. Bass The degree of polynomial growth of finitely generated nilpotent groups. Proc. London Math. Soc., 25 (1972), 603-614.

[14] I. Benjamini Instability of the Liouville property for quasi-isometric graphs and manifolds of polynomial volume growth. J. Theoret. Probab. 4 (1991), 631-637.

[15] I. Benjamini and J. Cao Examples of simply connected Liouville manifolds with positive spectrum. Differential Geom. Appl. 6 (1996), 31-50.

[16] D. Blackwell On transient markov processes with a countable number of states and stationary transition probabilities. Ann. Math. Stat. 26 (1955), 654-658.

[17] P. Bougerol and L. Elie Existence of positive harmonic functions on groups and on covering manifolds. Ann. Inst. H. Poincaré 31 (1995), 54-80.

[18] R. Brooks The fundamental group and the spectrum of the Laplacian. Comment. Math. Helv. 56 (1981), 581-598.

[19] K. Brown and R. Geoghegan An infinite dimensional torsion free $F P_{\infty}$ group. Invent. Math. 77 (1984), 367-381.

[20] A. Carey, T. Coulhon, V. Mathai, J. Phillips Von Neumann spectra near the spectral gap. Bull. Sci. Math. 122 (1998), 203-242.

[21] G. Carron Inégalités isopérimétriques de Faber-Krahn et conséquences. In Actes de la table ronde de géométrie différentielle (Luminy, France, 1992), Séminaires et Congrés 1, Soc. Math. France, 1996, pp. 205-232.

[22] I. Chavel Riemannian geometry: a modern introduction. Cambridge University Press, 1993.

[23] I. Chavel Isoperimetric inequalities. Differential geometric and analytic perspectives. Cambridge Tracts in Mathematics, 145. Cambridge University Press, Cambridge, 2001.

[24] S.Y. Cheng and S-T. Yau Differential equations on Riemannian manifolds and their geometric applications. Comm. Pure Appl. Math. 28 (1975), 333-354.

[25] G. Choquet and J. Deny Sur l'équation de convolution $\mu=\mu * \sigma$ C. R. Acad. Sci. Paris 250 (1960), 799-801.

[26] T. Colding and W. Minicozzi Harmonic functions on manifolds. Ann. of Math. 146 (1997), $725-747$.

[27] T. Coulhon Ultracontractivity and Nash type inequalities. J. Funct. Anal. 141 (1996), 510539.

[28] T. Coulhon Noyau de la chaleur et discrétisation d'une variété riemannienne. Israel J. Math. 80 (1992), 289-300. 
[29] T. Coulhon Analysis on infinite graphs with regular volume growth. In "Random walks and discrete potential theory, Cortona 1997" Sympos. Math. XXXIX (1999), 165-187, Cambridge University Press.

[30] T. Coulhon Heat kernel and isoperimetry on non-compact Riemannian manifolds. Contemporary Math. to appear.

[31] T. Coulhon and A. Grigor'yan On-diagonal lower bounds for heat kernels and Markov chains. Duke Math. J 89 (1997), 133-199.

[32] T. Coulhon, A. Grigor'yan and C. Pittet A geometric approach to on diagonal heat kernel lower bounds on groups. Ann. Inst. Fourier 51 (2001), 1763-1827.

[33] T. Coulhon, I. Holopainen, L. Saloff-Coste Harnack inequality and hyperbolicity for subelliptic p-Laplacians with applications to Picard type theorems. Geom. Funct. Anal. 11 (2001), 1139-1191.

[34] T. Coulhon and M. Ledoux Isopérimétrie, décroissance du noyau de la chaleur et transformations de Riesz: un contre exemple. Ark. Mat. 32 (1994), 63-77.

[35] T. Coulhon and L. Saloff-Coste Isopérimétrie sur les groupes et les variétés. Rev. Math. Iberoamericana 9 (1993), 293-314.

[36] T. Coulhon and L. Saloff-Coste Variétés riemanniennes isométriques à l'infini. Rev. Mat. Iberoamericana 13 (1995), 687-726.

[37] E.B. Davies Non-Gaussian aspects of heat kernel behaviour. J. London Math. Soc. 55 (1997), $105-125$.

[38] T. Delmotte Parabolic Harnack inequality and estimates of Markov chains on graphs. Rev. Mat. Iberoamericana 15 (1999), 181-232.

[39] J. Deny Méthodes hilbertiennes en théorie du potentiel. C.I.M.E., 1 ciclo, Stresa 1969 (1970), 121-201, Edizioni Cremonese, Rome.

[40] J. Dodziuk Difference equations, isoperimetric inequality and transience of certain random walks. Trans. Amer. Math. Soc. 284 (1984), 787-794.

[41] J. Dodziuk and V. Mathai Approximating $L^{2}$ invariants of amenable covering spaces: a combinatorial approach. J. Funct. Anal. 154 (1998), 359-378.

[42] N. Dungey Heat kernel estimates and Riesz transforms on some Riemannian covering manifolds. To appear in Math. Zeit.

[43] E. Dynkin and M. Maljutov Random walks on roups with a finite number of generators. (Russian) Dokl. Akad. Nauk SSSR 137 (1961), 1042-1045.

[44] V. A. Efremovic The proximity geometry of Riemannian manifolds. Uspekhi Math. Nauk. 8 (1953), 189.

[45] A. Erschler (Dyubina) On isoperimetric profiles of finitely generated groups. Geometriae Dedicata 100 (2003), 157-171.

[46] A. Erschler (Dyubina) Liouville property for groups and manifolds. Invent. Math. 155 (2004), 55-80.

[47] A. Erschler (Dyubina) Isoperimetry for wreath products of Markov chains and multiplicity of the selfintersections of randowm walks. Preprint, 2004.

[48] E. Følner On groups with full Banach mean value. Math. Scand. 3 (1955), 243-254.

[49] H. Furstenberg Random walks and discrete subgroups of Lie groups. 1971 Advances in Probability and Related Topics, Vol. 1, 1-63 Dekker, New York.

[50] H. Furstenberg Boundary theory and stochastic processes on homogeneous spaces. In "Harmonic analysis on homogeneous spaces" (Proc. Sympos. Pure Math., Vol. XXVI, Williams Coll., Williamstown, Mass., 1972), 193-229. Amer. Math. Soc., Providence, R.I., 1973

[51] R. Grigorchuk Symmetrical random walks on discrete groups. In "Multicomponent random systems" (Dobrushin et al, eds), Advances in Probability and Related Topics 6 (1980), 285325, Dekker.

[52] R. Grigorchuk On growth in group theory. In "Proceeding of the International Congress of Mathematicians" (Kyoto, 1990), Math. Soc. Japan, 1991, 325-338.

[53] R. Grigorchuk and P. de la Harpe On problems related to growth, entropy, and spectrum in group theory. J. Dynam. Control Systems 3 (1997), 51-89.

[54] A. Grigor'yan The heat equation on non-compact Riemannian manifolds. Matem Sbornik 182 (1991), 55-87. Engl. Transl. Math. USSR Sb. 72 (1992), 47-77.

[55] A. Grigor'yan Heat kernel upper bounds on a complete non-compact manifold. Rev. Mat. Iberoamericana 10 (1994), 395-452. 
[56] A. Grigor'yan Analytic and geometric background of recurrence and non-explosion of the Brownian motion on Riemannian manifolds. Bull. Amer. Math. Soc. 36 (1999), 135-249.

[57] A. Grigor'yan and L. Saloff-Coste Heat kernel on connected sums of Riemannian manifolds. Math. Res. Letters 6 (1999), 307-321.

[58] M. Gromov Groups of polynomial growth and expanding maps. Publ. Math. I.H.E.S. 53 (1981), 53-73.

[59] M. Gromov Asymptotic invariants of infinite groups in Geometric Group Theory. LMS Lecture notes series 182(II), Cambridge University Press, 1993.

[60] M. Gromov Metric structures for Riemannian and non-Riemannian spaces. Birkhäuser, 1999.

[61] Y. Guivarc'h Croissance polynômiale et périodes des fonctions harmoniques. Bull. Soc. Math. France 101 (1973), 333-379.

[62] Y. Guivarc'h Application d'un théorème limite local à la transience et à la récurrence de marches de Markov. In "Théorie du Potentiel” Lecture Notes in Math. 1096 (1984), 301-332, Springer.

[63] P. de la Harpe Topics on geometric group theory. Chicago Lectures in Mathematics. University of Chicago Press, 2000.

[64] W. Hebisch and L. Saloff-Coste Gaussian estimates for Markov chains and random walks on groups. Ann. Prob. 21 (1993), 673-709.

[65] J. Heinonen and P. Koskela Quasiconformal maps in metric spaces with controlled geometry. Acta Math. 181 (1998), 1-61.

[66] I. Holopainen and P. Soardi p-harmonic functions on graphs and manifolds. Manuscripta Math. 94 (1997), 95-110.

[67] V. Kaimanovich Brownian motion and harmonic functions on covering manifolds. An entropic approach. (Russian) Dokl. Akad. Nauk SSSR 288 (1986), 1045-1049.

[68] V. Kaimanovich Boundaries of random walks on polycyclic groups and the law of large numbers for solvable Lie groups. Vestnik Leningrad University: Math. 20 (1987), 49-52.

[69] V. Kaimanovich Discretization of bounded harmonic functions on Riemannian manifolds and entropy. In "Proceedings of the International Conference on Potential Theory, Nagoya" (M. Kishi, ed.), de Gruyter (1992), 213-223.

[70] V. Kaimanovich Dirichlet norms, capacities and generalized isoperimetric inequalities for Markov operators. Pot. Anal. 1 (1992), 61-82.

[71] V. Kaimanovich Poisson boundaries of random walks on discrete solvable groups. In "Probability measures on groups X" (Oberwolfach 1990, H. Heyer, ed) (1991), 205-238, Plenum.

[72] V. Kaimanovich and A. Vershik Random walks on discrete groups: boundary and entropy Ann. Probab. 11 (1983), 457-490.

[73] M. Kanai Rough isometries and the parabolicity of Riemannian manifolds. J. Math. Soc. Japan 38 (1986), 227-238.

[74] M. Kanai Rough isometries and the combinatorial approximations of geometries of noncompact Riemannian manifolds. J. Math. Soc. Japan 37 (1985), 391-413.

[75] M. Krasnoselsky and Y. Rutitsky Convex functions and Orlicz spaces. Noordhof, 1961.

[76] H. Kesten Symmetric random walks on groups. Trans. Amer. Math. Soc. 92 (1959), 336-354.

[77] H. Kesten Full Banach mean values on countable groups. Math. Scand. 7 (1959), 146-156.

[78] G. Lawler Intersections of random walks Bikhäuser, 1991.

[79] P. Li Curvature and function theory on Riemannian manifolds. In "Surveys in Differential Geometry, VII" International Press (2000), 375-432.

[80] P. Li and S-T. Yau On the parabolic kernel of the Schrödinger operator Acta Math. 156 (1986), 153-201.

[81] J. Lott Heat kernels on covering spaces and topological invariants J. Diff. Geom. 35 (1992), $471-510$

[82] J. Lott Remarks about heat diffusion on periodic spaces. Proc. Amer. Math. Soc. 127 (1997), 1243-1249.

[83] T. Lyons A simple criterion for transience of a reversible Markov chain. Ann. Probab. 11 (1983), 393-402.

[84] T. Lyons Instability of the Liouville property for quasi-isometric Riemannian manifolds and reversible Markov chains. J. Diff. Geom. 26 (1981), 33-66.

[85] T. Lyons Random thoughts on reversible potential theory. In "Summer School in Potential Theory, Joensuu 1990" (I. Laine, ed), University of Joensuu (1992), 71-114. 
[86] T. Lyons and D. Sullivan Function theory, random paths and covering spaces. J. Diff. Geom. 19 (1984), 299-323.

[87] S. Maillot Large-scale conformal rigidity in dimension three. Preprint, 2002.

[88] G. Margulis Positive harmonic functions on nilpotent groups. (Russian) Dokl Akad. Nauk SSSR 166 (1966), 1054-1057. Engl. Transl. Soviet Math. Dokl. 7 (1966), 241-244.

[89] V. Maz'ja Sobolev Spaces. Springer, 1985.

[90] J. Milnor Growth in finitely generated solvable groups. J. Diff. Geom. 2 (1968), 447-449.

[91] B. Morris and Y. Peres Evolving sets, mixing and heat kernel bounds. Probab. Theory Related Fields, to appear.

[92] G.D. Mostov Fundamental groups of homogeneous spaces. Ann. of Math. 60 (1954), 1-27.

[93] Nash J. Continuity of solutions of parabolic and elliptic equations. American Math. J. 80 (1958), 931-954.

[94] P. Pansu Profil isopérimétrique, métriques périodiques et formes d'équilibre des cristaux. ESAIM Control Optim. Calc. Var. 4 (1999), 631-665

[95] A. L. Paterson Amenability. Mathematical Surveys and Monographs, Vol. 29. American Mathematical Society, 1988.

[96] Ch. Pittet Følner sequences on polycyclic groups. Rev. Math. Iberoamericana 11 (1995), 675-685.

[97] Ch. Pittet The isoperimetric profile of homogeneous Riemannian manifolds. J. Diff. Geom. 54 (2000), 255-302.

[98] Ch. Pittet and L. Saloff-Coste Isoperimetry and random walk on discrete subgroups of connected Lie groups. In Random Walk and Discrete Potential Theory, Cortona, (M. Picardello and W. Woess, eds.) Cambridge University Press, 1999, pp 306-319.

[99] Ch. Pittet and L. Saloff-Coste Amenable groups, isoperimetric profile and random walks. In "Geometric Group Theory Down Under" (J. Cossey, Ch. F. Miller III, W. Neumann, and M. Shapiro, eds.), Walter de Gruyter, 1999, pp. 293-316.

[100] Ch. Pittet and L. Saloff-Coste On the stability of the behavior of random walks on groups. J.Geom. Anal. 10 (2001), 701-726.

[101] Ch. Pittet and L. Saloff-Coste On random walks on wreath products. Ann. Probab. 30 (2002), 948-977.

[102] Ch. Pittet and L. Saloff-Coste Random walks on abelian-by-cyclic groups. Proc. Amer. Math. Soc. 131 (2002), 1071-1079.

[103] Ch. Pittet and L. Saloff-Coste Random walks on finite rank solvable groups. J. Europ. Math. Soc. 5 (2003), 313-342.

[104] Ch. Pittet and L. Saloff-Coste A survey on the relationships between volume growth, isoperimetry, and the behavior of simple random walks on Cayley graphs, with examples. Unpublished manuscript.

[105] M.S. Raghunathan Discrete subgroups of Lie groups. Ergebnisse der Mathematik und ihrer Grenzgebiete, Band 68. Springer, 1972.

[106] [43] D. W. Robinson Elliptic operators and Lie groups. Oxford Mathematical Monographs. Oxford University Press, New York, 1991.

[107] D.J.S. Robinson A Course in the Theory of Groups. Graduate texts in Mathematics, Springer, 1993.

[108] H. Royden On the ideal boundary of a Riemann surface. In "Contributions to the theory of Riemann surfaces" Annals of Mathematics Studies, no. 30 (1953), 107-109, Princeton University Press.

[109] L. Saloff-Coste A note on Poincaré, Sobolev and Harnack inequalities. Duke Math. J. 65 (1992), IMRN 27-38.

[110] L. Saloff-Coste On global Sobolev inequalities. Forum Math. 6 (1994), 271-286.

[111] L. Saloff-Coste Aspects of Sobolev Inequalities. Cambridge university Press, 2001.

[112] L. Saloff-Coste and W. Woess Transition operators on co-compact G-spaces. Preprint (2003).

[113] A. S. Schwarzc A volume invariant of coverings (In Russian) Dokl. Ak. Nauk. 105 (1955), 32-34.

[114] F. Spitzer Principles of Random Walk. Van Nostrand, (1972).

[115] P. Soardi Potential theory on infinite networks. Lect. Notes in Math. 1590. Springer, 1994.

[116] W. Thurston Three-Dimensional Geometry and Topology. Princeton University Press, 1997.

[117] J. Tits Free subgroups in linear groups. J. Algebra, 20 (1972), 250-270. 
[118] L. Van den Dries and A. Wilkie Gromov's theorem on groups of polynomial growth and elementary logic. J. Alg. 89 (1984), 349-374.

[119] N. Varopoulos Brownian motion and transient groups. Ann. Inst. Fourier 33 (1983), 241261.

[120] N. Varopoulos Brownian motion and random walks on manifolds. Ann. Inst. Fourier 34 (1984), 243-269.

[121] N. Varopoulos Isoperimetric inequalities and Markov chains. J. Funct. Anal. 63 (1985), $215-239$.

[122] N. Varopoulos Théorie du potentiel sur les groupes nilpotents. C. R. Acad. Sci. Paris Sr. I Math. 301 (1985), 143-144.

[123] N. Varopoulos Théorie du potentiel sur des groupes et des variétés. C. R. Acad. Sci. Paris Sr. I Math. 302 (1986), 203-205.

[124] N. Varopoulos, Analysis on nilpotent groups. J. Funct. Anal. 66 (1986), 406-431.

[125] N. Varopoulos Random walks and Brownian motion on manifolds. In "Harmonic Analysis, Symmetric Spaces and Probability Theory, Cortona 1984" Sympos. Math. XXIX (1987), 97109, Academic Press.

[126] N. Varopoulos Groups of superpolynomial growth. In "Harmonic analysis (Sendai, 1990)", 194-200, ICM-90 Satell. Conf. Proc., Springer, Tokyo, 1991.

[127] N. Varopoulos Analysis and geometry on groups. In "Proceeding of the International Congress of Mathematicians" (Kyoto, 1990), Math. Soc. Japan, 1991, 951-957..

[128] N. Varopoulos, L. Saloff-Coste and T. Coulhon Analysis and geometry on groups. Cambridge University Press, (1993).

[129] A. M. Vershik Dynamic theory of growth in groups: entropy, boundaries, examples. (Russian. Russian summary) Uspekhi Mat. Nauk 55 (2000), 59-128; translation in Russian Math. Surveys 55 (2000), 667-733.

[130] A. M. Vershik Geometry and dynamics on the free solvable groups. arXiv:math.GR/0006177, June 2000.

[131] W. Woess Random walks on infinite graphs and groups - A survey on selected topics. Bull. London Math. Soc. 26 (1994), 1-60.

[132] W. Woess Random walks on infinite graphs and groups. Cambridge University Press, 2000.

[133] J. Wolf Growth of finitely generated solvable groups and curvature of Riemannian manifolds. J. Diff. Geom. 2 (1968), 424-446.

[134] S-T. Yau Harmonic functions on complete Riemannian manifolds Comm. Pure Appl. Math. 28 (1975), 201-228.

Department of Mathematics, Cornell University, Ithaca NY 14853

E-mail address: lsc@math.cornell.edu 